\title{
On exceptional quotient singularities
}

\author{
IVAN CHELTSOV \\ CONSTANTIN SHRAMOV
}

\begin{abstract}
We study exceptional quotient singularities. In particular, we prove an exceptionality criterion in terms of the $\alpha$-invariant of Tian, and utilize it to classify four-dimensional and five-dimensional exceptional quotient singularities.
\end{abstract}

We assume that all varieties are projective, normal, and defined over $\mathbb{C}$.

\section{Introduction}

Let $X$ be a smooth Fano variety (see Iskovskikh and Prokhorov [19]) of dimension $n$, and let $g=g_{i \bar{j}}$ be a Kähler metric with a Kähler form

$$
\omega=\frac{\sqrt{-1}}{2 \pi} \sum g_{i \bar{j}} d z_{i} \wedge d \bar{z}_{j} \in \mathrm{c}_{1}(X) .
$$

Definition 1.1 The metric $g$ is a Kähler-Einstein metric if $\operatorname{Ric}(\omega)=\omega$, where $\operatorname{Ric}(\omega)$ is a Ricci curvature of the metric $g$.

Let $\bar{G} \subset \operatorname{Aut}(X)$ be a compact subgroup. Suppose that $g$ is $\bar{G}$-invariant.

Definition 1.2 Let $P_{\bar{G}}(X, g)$ be the set of $C^{2}$-smooth $\bar{G}$-invariant functions $\varphi$ such that

$$
\omega+\frac{\sqrt{-1}}{2 \pi} \partial \bar{\partial} \varphi>0
$$

and $\sup _{X} \varphi=0$. Then the $\bar{G}$-invariant $\alpha$-invariant of the variety $X$ is the number

$$
\alpha_{\bar{G}}(X)=\sup \left\{\lambda \in \mathbb{Q} \mid \exists C \in \mathbb{R} \text { such that } \int_{X} e^{-\lambda \varphi} \omega^{n} \leqslant C \text { for any } \varphi \in P_{\bar{G}}(X, g)\right\} \text {. }
$$

The number $\alpha_{\bar{G}}(X)$ was introduced by Tian [42] and Tian and Yau [44] and now it is called the $\alpha$-invariant of Tian. 
Theorem 1.3 [42] The Fano variety $X$ admits a $\bar{G}$-invariant Kähler-Einstein metric if $\alpha_{\bar{G}}(X)>n /(n+1)$.

The normalized Kähler-Ricci flow on the smooth Fano $X$ is defined by the equation

$$
\left\{\begin{array}{l}
\frac{\partial \omega(t)}{\partial t}=-\operatorname{Ric}(\omega(t))+\omega(t), \\
\omega(0)=\omega,
\end{array}\right.
$$

where $\omega(t)$ is a Kähler form such that $\omega(t) \in \mathrm{c}_{1}(X)$, and $t \in \mathbb{R}_{\geqslant 0}$. It follows from Cao [8] that the solution $\omega(t)$ to (1.4) exists for every $t>0$.

Theorem 1.5 (Tian-Zhu [45]) If $X$ admits a Kähler-Einstein metric with a Kähler form $\omega_{\mathrm{KE}}$, then any solution to (1.4) converges to $\omega_{\mathrm{KE}}$ in the sense of Cheeger-Gromov.

The normalized Kähler-Ricci iteration on the smooth Fano variety $X$ is defined by the equation

$$
\left\{\begin{array}{l}
\omega_{i-1}=\operatorname{Ric}\left(\omega_{i}\right) \\
\omega_{0}=\omega
\end{array}\right.
$$

where $\omega_{i}$ is a Kähler form such that $\omega_{i} \in \mathrm{c}_{1}(X)$. It follows from Yau [46] that the solution $\omega_{i}$ to (1.6) exists for every $i \geqslant 1$.

Theorem 1.7 (Rubinstein [35]) If $\alpha_{\bar{G}}(X)>1$ then $X$ admits a $\bar{G}$-invariant KählerEinstein metric with a Kähler form $\omega_{\mathrm{KE}}$ and any solution to (1.6) converges to $\omega_{\mathrm{KE}}$ in $C^{\infty}(X)$-topology.

Smooth Fano varieties that satisfy all hypotheses of Theorem 1.7 do exist.

Example 1.8 Let $X$ be a smooth del Pezzo surface such that $K_{X}^{2}=5$. Then $X$ is unique and $\operatorname{Aut}(X) \cong \mathrm{S}_{5}$. Moreover, one can show that $\alpha_{\bar{G}}(X)=2$ in the case when $\bar{G} \cong \mathrm{S}_{5}$ or $\bar{G} \cong \mathrm{A}_{5}$ (see Cheltsov [9, Example 1.11] and Cheltsov and Shramov [11, Theorem A.3]).

Suppose now that $X=\mathbb{P}^{n}$ (the simplest possible case). Then the Fubini-Study metric on $\mathbb{P}^{n}$ is Kähler-Einstein. Moreover, if $\bar{G}$ is the maximal compact subgroup of Aut $\left(\mathbb{P}^{n}\right)$, then the only $\bar{G}$-invariant metric on $\mathbb{P}^{n}$ is the Fubini-Study metric and we have $\alpha_{\bar{G}}\left(\mathbb{P}^{n}\right)=+\infty$ by Definition 1.2. In particular, the solution to (1.6) is trivial (and constant) in the latter case, since the initial metric $g$ must be the Fubini-Study metric. On the other hand, the convergence of any solution to (1.6) is not clear in the case when $\bar{G}$ is a finite group. So, Yanir Rubinstein asked the following question in the spring of 2009. 
Question 1.9 Is there a finite subgroup $\bar{G} \subset \operatorname{Aut}\left(\mathbb{P}^{n}\right)$ such that $\alpha_{\bar{G}}\left(\mathbb{P}^{n}\right)>1$ ?

This paper is inspired by Question 1.9. In particular, we will show that the answer to Question 1.9 is positive in the case when $n \leqslant 4$, which follows from [11, Theorem A.3] and Theorems 4.1, 4.2, 4.13, 5.6 and 3.21.

It came as a surprise that Question 1.9 is strongly related to the notion of exceptional singularity that was introduced by Vyacheslav Shokurov in [39]. Let us recall this notion. Let $(V \ni O)$ be a germ of Kawamata log terminal singularity (see Kollár [23, Definition 3.5]).

Definition 1.10 [39, Definition 1.5] The singularity $(V \ni O)$ is said to be exceptional if for every effective $\mathbb{Q}$-divisor $D_{V}$ on the variety $V$ such that $\left(V, D_{V}\right)$ is $\log$ canonical (see [23, Definition 3.5]) and for every resolution of singularities $\pi: U \rightarrow V$ there exists at most one $\pi$-exceptional divisor $E \subset U$ such that $a\left(V, D_{V}, E\right)=-1$, where the rational number $a\left(V, D_{V}, E\right)$ can be defined through the equivalence

$$
K_{U}+D_{U} \sim \mathbb{Q} \pi^{*}\left(K_{V}+D_{V}\right)+\sum a\left(V, D_{V}, E\right) E,
$$

where the sum is taken over all $f$-exceptional divisors, and $D_{U}$ is the proper transform of the divisor $D_{V}$ on the variety $U$.

One can show that exceptional Kawamata log terminal singularities are straightforward generalizations of the Du Val singularities of type $\mathbb{E}_{6}, \mathbb{E}_{7}$ and $\mathbb{E}_{8}$ (cf Theorem 4.1), which partially justifies the word "exceptional" in Definition 1.10.

Remark 1.11 One can easily check (for example, by applying Theorem 3.11) that the singularity $(V \ni O)$ is not exceptional if $V$ is smooth and $\operatorname{dim}(V) \geqslant 2$.

It follows from Shokurov [38], Ishii and Prokhorov [18] and Markushevich and Prokhorov [27] that exceptional Kawamata log terminal singularities do exist in dimensions 2 and 3. The existence in dimension 4 follows from Johnson and Kollár [20] and Prokhorov [31, Theorem 4.9]. Actually, exceptional Kawamata log terminal singularities exist in every dimension (see Example 3.13). We will see later (cf Theorem 1.14, Remark 1.16, Theorem 1.17 and Conjecture 1.23) that Question 1.9 is almost equivalent to the following

Question 1.12 Are there exceptional quotient singularities of dimension $n+1$ ? 
Recall that quotient singularities are always Kawamata log terminal by [23, Proposition 3.16]. So Question 1.12 fits well to Definition 1.10. Moreover, it follows from Shokurov [39] and Markushevich and Prokhorov [27] that the answer to Question 1.12 is positive for $n=1$ and $n=2$, respectively. The purpose of this paper is to study exceptional quotient singularities and, in particular, to give positive answers to Questions 1.9 and 1.12 for every $n \leqslant 4$. In a subsequent paper we will show that the answers to Questions 1.9 and 1.12 are still positive for $n=5$ and are surprisingly negative for $n=6$ (see [10]). So it is hard to predict what would be the answer to Question 1.9 in general. However, we still believe in the following:

Conjecture 1.13 For every $N \in \mathbb{Z}_{>0}$ there exist exceptional quotient singularities of dimension greater than $N$.

Let $G$ be a finite subgroup in $\mathrm{GL}_{n+1}(\mathbb{C})$, where $n \geqslant 1$. Denote by $Z(G)$ the center and by $[G, G]$ the commutator of group $G$. Let $\phi: \mathrm{GL}_{n+1}(\mathbb{C}) \rightarrow \operatorname{Aut}\left(\mathbb{P}^{n}\right) \cong \mathrm{PGL}_{n+1}(\mathbb{C})$ be the natural projection. Put $\bar{G}=\phi(G)$ and put $\operatorname{lct}\left(\mathbb{P}^{n}, \bar{G}\right)=\sup \left\{\begin{array}{l|l}\lambda \in \mathbb{Q} & \begin{array}{l}\text { the log pair }\left(\mathbb{P}^{n}, \lambda D\right) \text { has log canonical singularities } \\ \text { for every } \bar{G} \text {-invariant effective } \mathbb{Q} \text {-divisor } D \sim_{\mathbb{Q}}-K_{\mathbb{P}^{n}}\end{array}\end{array}\right\}$.

Theorem 1.14 (See eg [11, Theorem A.3].) One has $\operatorname{lct}\left(\mathbb{P}^{n}, \bar{G}\right)=\alpha_{\bar{G}}\left(\mathbb{P}^{n}\right)$.

The number $\operatorname{lct}\left(\mathbb{P}^{n}, \bar{G}\right)$ is usually called $\bar{G}$-equivariant global log canonical threshold of $\mathbb{P}^{n}$. Despite the fact that $\operatorname{lct}\left(\mathbb{P}^{n}, \bar{G}\right)=\alpha_{\bar{G}}\left(\mathbb{P}^{n}\right)$, we still prefer to work with the number $\operatorname{lct}\left(\mathbb{P}^{n}, \bar{G}\right)$ throughout this paper, because it is easier to handle than $\alpha_{\bar{G}}\left(\mathbb{P}^{n}\right)$. For example, it follows immediately from Definition 3.1 that $\operatorname{lct}\left(\mathbb{P}^{n}, \bar{G}\right) \leqslant d /(n+1)$ if the group $G$ has a semi-invariant of degree $d$ (a semi-invariant of the group $G$ is a polynomial whose zeroes define a $\bar{G}$-invariant hypersurface in $\mathbb{P}^{n}$ ).

Remark 1.15 A semi-invariant of the group $G$ is its invariant if $Z(G) \subseteq[G, G]$ and $\bar{G}$ is a nonabelian simple group.

Recall that an element $g \in G$ is called a reflection (or sometimes a quasireflection) if there is a hyperplane in $\mathbb{P}^{n}$ that is pointwise fixed by $\phi(g)$ (cf Springer [40, Section 4.1]).

Remark 1.16 Let $R \subseteq G$ be a subgroup generated by all reflections. Then the quotient $\mathbb{C}^{n+1} / R$ is isomorphic to $\mathbb{C}^{n+1}$ (see Shephard and Todd [37] and Springer [40, Theorem 4.2.5]). Moreover, the subgroup $R \subseteq G$ is normal, and the singularity $\mathbb{C}^{n+1} / G$ is isomorphic to the singularity $\mathbb{C}^{n+1} /(G / R)$. Note that the subgroup $R$ is trivial if $G \subset \mathrm{SL}_{n+1}(\mathbb{C})$. If $G$ is a trivial group, then the singularity $\mathbb{C}^{n+1} / G \cong \mathbb{C}^{n+1}$ is not exceptional by Remark 1.11 . 
Thus to answer Question 1.12 one can always assume that the group $G$ does not contain reflections. On the other hand, one can easily check that there exists a finite subgroup $G^{\prime} \subset \mathrm{SL}_{n+1}(\mathbb{C})$ such that $\phi\left(G^{\prime}\right)=\bar{G}$. So to answer Question 1.9 one can also assume that $G \subset \mathrm{SL}_{n+1}(\mathbb{C})$, which implies, in particular, that the group $G$ does not contain reflections. Moreover, if the group $G$ does not contain reflections, then the singularity $\mathbb{C}^{n+1} / G$ is exceptional if and only if the singularity $\mathbb{C}^{n+1} / G^{\prime}$ is exceptional thanks to the following:

Theorem 1.17 Let $G$ be a finite subgroup in $\mathrm{GL}_{n+1}(\mathbb{C})$ that does not contain reflections. Then

- the singularity $\mathbb{C}^{n+1} / G$ is exceptional if $\operatorname{lct}\left(\mathbb{P}^{n}, \bar{G}\right)>1$,

- the singularity $\mathbb{C}^{n+1} / G$ is not exceptional if either $\operatorname{lct}\left(\mathbb{P}^{n}, \bar{G}\right)<1$ or $G$ has a semi-invariant of degree at most $n+1$,

- for any subgroup $G^{\prime} \subset \mathrm{GL}_{n+1}(\mathbb{C})$ such that $G^{\prime}$ does not contain reflections and $\phi\left(G^{\prime}\right)=\bar{G}$, the singularity $\mathbb{C}^{n+1} / G$ is exceptional if and only if the singularity $\mathbb{C}^{n+1} / G^{\prime}$ is exceptional.

Proof All required assertions immediately follow from Theorem 3.17 (cf [32, Proposition 3.1; 32, Lemma 3.1]).

It should be pointed out that the assumption that $G$ contains no reflections is crucial for Theorem 1.17.

Example 1.18 Let $G$ be a finite subgroup in $\mathrm{GL}_{4}(\mathbb{C})$ that is the subgroup number 32 in Shephard and Todd [37, Table VII]. Then the group $G$ is generated by reflections (see [37]), so that the singularity $\mathbb{C}^{4} / G$ is not exceptional by Remark 1.16 . On the other hand, it follows from Theorem 4.13 that $\operatorname{lct}\left(\mathbb{P}^{3}, \bar{G}\right) \geqslant 5 / 4$, because $\bar{G} \cong \operatorname{PSp}_{4}\left(\mathbb{F}_{3}\right)$. It follows from Theorem 4.13 that there exists a subgroup $G^{\prime} \subset \mathrm{SL}_{4}(\mathbb{C})$ such that $\bar{G}=\phi\left(G^{\prime}\right)$ and the singularity $\mathbb{C}^{4} / G^{\prime}$ is exceptional. One can produce similar examples for two-dimensional and three-dimensional singularities.

By Theorem 1.17 and [40, Section 4.5], if $G$ is a finite subgroup in $\mathrm{GL}_{2}(\mathbb{C})$ that does not contain reflections, then the singularity $\mathbb{C}^{2} / G$ is exceptional if and only if $G$ has no semi-invariants of degree at most 2 . A similar result holds in dimension 3 .

Theorem 1.19 [27, Theorem 1.2] Let $G$ be a finite group in $\mathrm{GL}_{3}(\mathbb{C})$ that does not contain reflections. Then the singularity $\mathbb{C}^{3} / G$ is exceptional if and only if $G$ does not have semi-invariants of degree at most 3 . 
For finite subgroups in $\mathrm{GL}_{4}(\mathbb{C})$, the assertion of Theorem 1.19 is no longer true.

Example 1.20 [32, Example 3.1] Let $\Gamma \subset \mathrm{SL}_{2}(\mathbb{C})$ be a binary icosahedron group. Put

$$
G=\left\{\left(\begin{array}{cccc}
a_{11} & a_{12} & 0 & 0 \\
a_{21} & a_{22} & 0 & 0 \\
0 & 0 & b_{11} & b_{12} \\
0 & 0 & b_{21} & b_{22}
\end{array}\right) \mid\left(\begin{array}{ll}
a_{11} & a_{12} \\
a_{21} & a_{22}
\end{array}\right) \in \Gamma \ni\left(\begin{array}{ll}
b_{11} & b_{12} \\
b_{21} & b_{22}
\end{array}\right)\right\} \subset \mathrm{SL}_{4}(\mathbb{C}),
$$

where $a_{i j} \in \mathbb{C} \ni b_{i j}$. Then $G$ does not have semi-invariants of degree at most 4 , because $\Gamma$ does not have semi-invariants of degree at most 4 (see [40, Section 4.5]). On the other hand, it follows from [32, Proposition 2.1] that the singularity $\mathbb{C}^{4} / G$ is not exceptional (cf Corollary 3.20).

Actually, it is possible to modify the assertion of Theorem 1.19 so that its new version can be generalized to higher dimensions.

Definition 1.21 (Blichfeldt [3]) The subgroup $G \subset \mathrm{GL}_{n+1}(\mathbb{C})$ is said to be primitive if there is no nontrivial decomposition $\mathbb{C}^{n+1}=\bigoplus_{i=1}^{r} V_{i}$ such that for any $g \in G$ and any $i$ there is some $j=j(g)$ such that $g\left(V_{i}\right)=V_{j}$.

If $G$ is primitive, then $\bar{G} \cong G / Z(G)$ by Schur's lemma. It follows from [32, Proposition 2.1] that $G$ must be primitive if $\mathbb{C}^{n+1} / G$ is exceptional (we give a short proof of this fact in Corollary 3.20). Moreover, primitivity plays a crucial role in the main result of this paper:

Theorem 1.22 Let $G$ be a finite subgroup in $\mathrm{GL}_{n+1}(\mathbb{C})$ that does not contain reflections. Suppose that $n \leqslant 4$. Then the following conditions are equivalent:

- The singularity $\mathbb{C}^{n+1} / G$ is exceptional.

- $\operatorname{lct}\left(\mathbb{P}^{n}, \bar{G}\right) \geqslant(n+2) /(n+1)$.

- The group $G$ is primitive and has no semi-invariants of degree at most $n+1$.

Proof The required assertion follows from Theorems 1.19, 3.17, 3.18, 3.21, 4.13 and 5.6.

It appears that in higher dimensions exceptionality cannot be expressed in terms of primitivity and absence of semi-invariants of small degree. In particular, there are nonexceptional six-dimensional quotient singularities arising from primitive subgroups without reflections in $\mathrm{GL}_{6}(\mathbb{C})$ that have no semi-invariants of degree at most 6 (see 
Example 3.25). On the other hand, it follows from Theorem 1.22 that we may expect the sufficient condition for exceptionality in Theorem 1.17 to be a necessary condition as well. Namely, inspired by Theorem 1.22 and Tian [43, Question 1] we believe in the following:

Conjecture 1.23 Let $G$ be a finite subgroup in $\mathrm{GL}_{n+1}(\mathbb{C})$ that does not contain reflections. Then the singularity $\mathbb{C}^{n+1} / G$ is exceptional if and only if $\operatorname{lct}\left(\mathbb{P}^{n}, \bar{G}\right)>1$.

It follows from Theorem 1.22 that Conjecture 1.23 holds for $n \leqslant 4$. In a subsequent paper we will show that Conjecture 1.23 holds for $n=5$ and $n=6$ (see [10]). Note that Conjecture 1.23 is a special case of Conjecture 3.5.

To apply Theorem 1.22 we may assume that $G \subset \mathrm{SL}_{n+1}(\mathbb{C})$, since there exists a finite subgroup $G^{\prime} \subset \mathrm{SL}_{n+1}(\mathbb{C})$ such that $\phi\left(G^{\prime}\right)=\bar{G}$. On the other hand, it is well known that there are at most finitely many primitive finite subgroups in $\mathrm{SL}_{n+1}(\mathbb{C})$ up to conjugation (see Collins [12]). Primitive finite subgroups of $\mathrm{SL}_{2}(\mathbb{C})$ are group-theoretic counterparts of Platonic solids and each of them gives rise to an exceptional singularity (see Theorem 4.1). Primitive finite subgroups of $\mathrm{SL}_{3}(\mathbb{C})$ are classified by Blichfeldt in [3]. Prokhorov and Markushevich used Blichfeldt's classification in [27] to obtain an explicit classification of the subgroups in $\mathrm{SL}_{3}(\mathbb{C})$ corresponding to three-dimensional exceptional quotient singularities (see Theorem 4.2). For dimension 2 the same was done by Shokurov (see Theorem 4.1). Similar classification is possible in dimensions 4 and 5 , since primitive finite subgroups of $\mathrm{SL}_{4}(\mathbb{C})$ and $\mathrm{SL}_{5}(\mathbb{C})$ are classified by Blichfeldt [3] and Brauer [5], respectively. In fact, we obtain a complete list of finite subgroups in $\mathrm{SL}_{4}(\mathbb{C})$ and $\mathrm{SL}_{5}(\mathbb{C})$ that satisfy all hypotheses of Theorem 1.22 (see Theorems 4.13 and 5.6).

While the exceptionality of a quotient singularity $\mathbb{C}^{n+1} / G$ depends on a lower bound for a global $\log$ canonical threshold $\operatorname{lct}\left(\mathbb{P}^{n}, \bar{G}\right)$, it is interesting to find upper bounds for $\operatorname{lct}\left(\mathbb{P}^{n}, \bar{G}\right)$ as well. Using [40, Section $\left.4.5 ; 47\right]$ and a bit of direct computation, we see that it follows from Corollary 3.19 that

$$
\operatorname{lct}\left(\mathbb{P}^{n}, \bar{G}\right) \leqslant \begin{cases}6 & \text { if } n=1 \\ 2 & \text { if } n=2 \\ 3 & \text { if } n=3\end{cases}
$$

Theorem 1.24 The inequality $\operatorname{lct}\left(\mathbb{P}^{n}, \bar{G}\right) \leqslant 4(n+1)$ holds for every $n \geqslant 1$. Moreover, if $n \geqslant 23$, then $\operatorname{lct}\left(\mathbb{P}^{n}, \bar{G}\right) \leqslant 12(n+1) / 5$.

Proof Let $p$ be any prime number which does not divide $|G|$. Then $G$ has a semiinvariant of degree at most $(p-1)(n+1)$ by [41, Lemma 2]. Thus, it follows 
from Definition 3.1 that $\operatorname{lct}\left(\mathbb{P}^{n}, \bar{G}\right) \leqslant p-1$. On the other hand, it follows from the Bertrand's postulate (see Ramanujan [34]) that there is a prime number $p^{\prime}$ such that $2 n+3<p^{\prime}<2(2 n+3)$, which implies that $p^{\prime} \leqslant 4 n+5$. If $G$ is not primitive, then $\operatorname{lct}\left(\mathbb{P}^{n}, \bar{G}\right) \leqslant 1$ by Corollary 3.19. If $G$ is primitive, then $p^{\prime}$ does not divide $|G|$ by Feit and Thompson [15, Theorem 1], which completes the proof of the first assertion of the theorem. A similar argument with an additional use of Nagura [29] gives the second assertion for $n \geqslant 23$.

In fact, we expect the following to be true (cf [41]).

Conjecture 1.25 There exists a universal constant $C \in \mathbb{R}$ such that $\operatorname{lct}\left(\mathbb{P}^{n}, \bar{G}\right) \leqslant C$ for any finite subgroup $\bar{G} \subset \operatorname{Aut}\left(\mathbb{P}^{n}\right)$ and for any $n \geqslant 1$.

Let us describe the structure of the paper. In Section 2 we collect auxiliary results. In Section 3 we prove the exceptionality criterion for a singularity $\mathbb{C}^{n+1} / G$. In Section 4 we classify exceptional quotient singularities in dimension 4 (see Theorem 4.13). In Section 5 we classify exceptional quotient singularities in dimension 5 (see Theorem 5.6). In Appendix A we prove Corollary A.2 and Theorem A.9 that are used in Section 5.

Many of our results can be obtained by direct computations using the Atlas of finite groups [13].

Throughout the paper we use the following standard notation: the symbol $\mathbb{Z}_{n}$ denotes the cyclic group of order $n$, the symbol $\mathbb{F}_{n}$ denotes the finite field consisting of $n$ elements, the symbol $\mathrm{S}_{n}$ denotes the symmetric group of degree $n$, the symbol $\mathrm{A}_{n}$ denotes the alternating group of degree $n$, the symbols GL, PGL, SL, PSL, $\operatorname{Sp}_{4}\left(\mathbb{F}_{3}\right)$ and $\mathrm{PSp}_{4}\left(\mathbb{F}_{3}\right)$ denote the corresponding algebraic groups. The symbol $k . G$ denotes a central extension of a group $G$ with the center $\mathbb{Z}_{k}$ (this might be nonunique).

Acknowledgements We would like to thank I Arzhantsev, S Galkin, V Dotsenko, A Khoroshkin, S Loktev, D Pasechnik, V Popov, Yu Prokhorov, S Rybakov, L Rybnikov and V Vologodsky for very useful and fruitful discussions. We thank T Köppe for helping us access the classical German literature on Invariant Theory.

The authors were partially supported by AG Laboratory GU-HSE, RF government grant 11 11.G34.31.0023. The first author was partially supported by the grants NSF DMS-0701465 and EPSRC EP/E048412/1. The second author was partially supported by the grants RFFI 08-01-00395-a, RFFI 11-01-00185-a, RFFI 11-01-00336-a, N.Sh.1987.2008.1, N.Sh.-4713.2010.1 and EPSRC EP/E048412/1. 


\section{Preliminaries}

Throughout the paper we use the standard language of the singularities of pairs (see Kollár [23]). By strictly log canonical singularities we mean log canonical singularities that are not Kawamata log terminal (see [23, Definition 3.5]).

Let $X$ be a variety, let $B_{X}$ and $D_{X}$ be effective $\mathbb{Q}$-divisors on the variety $X$ such that the singularities of the log pair $\left(X, B_{X}\right)$ are Kawamata log terminal, and $K_{X}+B_{X}+D_{X}$ is a $\mathbb{Q}$-Cartier divisor. Let $Z \subseteq X$ be a closed nonempty subvariety.

Definition 2.1 The log canonical threshold of the boundary $D_{X}$ along $Z$ is $\mathrm{c}_{Z}\left(X, B_{X}, D_{X}\right)=\sup \left\{\lambda \in \mathbb{Q} \mid\right.$ the pair $\left(X, B_{X}+\lambda D_{X}\right)$ is log canonical along $\left.Z\right\}$.

Note that the log pair $\left(X, B_{X}+D_{X}\right)$ is Kawamata log terminal along $Z$ if and only if $\mathrm{c}_{Z}\left(X, B_{X}, D_{X}\right)>1$. For simplicity, we put $\mathrm{c}\left(X, B_{X}, D_{X}\right)=\mathrm{c}_{X}\left(X, B_{X}, D_{X}\right)$. We put $\mathrm{c}_{Z}\left(X, D_{X}\right)=\mathrm{c}_{Z}\left(X, B_{X}, D_{X}\right)$ in the case when $B_{X}=0$. For simplicity, we also put $\mathrm{c}\left(X, D_{X}\right)=\mathrm{c}_{X}\left(X, D_{X}\right)$.

Apart from some rare but important occasions (cf Section 3), we only need to consider the case when $B_{X}=0$. So from now on we assume that $B_{X}=0$.

Let $\pi: \bar{X} \rightarrow X$ be a birational morphism such that $\bar{X}$ is smooth. Then

$$
K_{\bar{X}}+D_{\bar{X}} \sim \mathbb{Q} \pi^{*}\left(K_{X}+D_{X}\right)+\sum_{i=1}^{m} d_{i} E_{i},
$$

where $D_{\bar{X}}$ is a proper transform of the divisor $D_{X}$ on the variety $\bar{X}, d_{i} \in \mathbb{Q}$, and $E_{i}$ is an exceptional divisor of the morphism $\pi$. Put $D_{\bar{X}}=\sum_{i=1}^{r} a_{i} \bar{D}_{i}$, where $a_{i} \in \mathbb{Q} \geqslant 0$, and $\bar{D}_{i}$ is a prime Weil divisor on $\bar{X}$. Suppose that $\sum_{i=1}^{r} \bar{D}_{i}+\sum_{i=1}^{m} E_{i}$ is a divisor with simple normal crossing. Put

$$
\mathcal{I}\left(X, D_{X}\right)=\pi_{*} \mathcal{O}_{\bar{X}}\left(\sum_{i=1}^{m}\left\lceil d_{i}\right\rceil E_{i}-\sum_{i=1}^{r}\left\lfloor a_{i}\right\rfloor \bar{D}_{i}\right),
$$

and let $\mathcal{L}\left(X, D_{X}\right)$ be a subscheme that corresponds to the ideal sheaf $\mathcal{I}\left(X, D_{X}\right)$ (the sheaf $\mathcal{I}\left(X, D_{X}\right)$ is an ideal sheaf, because $D_{X}$ is an effective divisor). Put $\operatorname{LCS}\left(X, D_{X}\right)=\operatorname{Supp}\left(\mathcal{L}\left(X, D_{X}\right)\right)$.

Remark 2.2 If $\left(X, D_{X}\right)$ is $\log$ canonical, then $\mathcal{L}\left(X, D_{X}\right)$ is reduced. 
The subscheme $\mathcal{L}\left(X, D_{X}\right)$ and locus $\operatorname{LCS}\left(X, D_{X}\right)$ were introduced by Shokurov [38]. They are called are called the subscheme of log canonical singularities of the $\log$ pair $\left(X, D_{X}\right)$ and the locus of $\log$ canonical singularities of the log pair $\left(X, D_{X}\right)$, respectively. Note that the ideal sheaf $\mathcal{I}\left(X, D_{X}\right)$ is also known as the multiplier ideal sheaf of the log pair $\left(X, D_{X}\right)$ (see Lazarsfeld [25]).

Theorem 2.3 [25, Theorem 9.4.8] Let $H$ be a nef and big $\mathbb{Q}$-divisor on $X$ such that $K_{X}+D_{X}+H \equiv D$ for some Cartier divisor $D$ on the variety $X$. Then

$$
H^{i}\left(\mathcal{I}\left(X, D_{X}\right) \otimes D\right)=0
$$

for every $i \geqslant 1$.

Corollary 2.4 [38, Lemma 5.7] Suppose that $-\left(K_{X}+D_{X}\right)$ is nef and big. Then the locus $\operatorname{LCS}\left(X, D_{X}\right)$ is connected.

Let $\mathbb{L} \mathbb{C S}\left(X, D_{X}\right)$ be the set that consists of all possible centers of log canonical singularities of the log pair $\left(X, D_{X}\right)$ (see [11, Definition 2.2]).

Remark 2.5 Let $\mathcal{H}$ be a linear system on the variety $X$ that has no base points. Put $Z \cap H=\sum_{i=1}^{k} Z_{i}$, where $H$ is a general divisor in $\mathcal{H}$, and $Z_{i}$ is an irreducible subvariety in $H$. Then $Z \in \mathbb{L} \mathbb{C S}\left(X, D_{X}\right)$ if and only if all subvarieties $Z_{1}, \ldots, Z_{k}$ are contained in the set $\mathbb{L} \mathbb{C S}\left(H,\left.D_{X}\right|_{H}\right)$.

If $Z \in \mathbb{L} \mathbb{C S}\left(X, D_{X}\right)$ and no proper subvariety of $Z$ is contained in $\mathbb{L} \mathbb{C S}\left(X, D_{X}\right)$, then $Z$ is said to be a minimal center in $\mathbb{L} \mathbb{C S}\left(X, D_{X}\right)$ or minimal center of log canonical singularities of the log pair $\left(X, D_{X}\right)$.

Lemma 2.6 (Kawamata [21, Proposition 1.5]) Suppose that $Z \in \mathbb{L} \mathbb{C S}\left(X, D_{X}\right)$ and $\left(X, D_{X}\right)$ is $\log$ canonical. Let $Z^{\prime}$ be a center in $\mathbb{L} \mathbb{C S}\left(X, D_{X}\right)$ such that $\varnothing \neq Z \cap Z^{\prime}=$ $\sum_{i=1}^{k} Z_{i}$, where $Z_{i} \subsetneq Z$ is an irreducible subvariety. Then $Z_{i} \in \mathbb{L} \mathbb{C S}\left(X, D_{X}\right)$ for every $i \in\{1, \ldots, k\}$.

Theorem 2.7 [22, Theorem 1] Suppose $Z \subset X$ is a minimal center in $\mathbb{L} \mathbb{C S}\left(X, D_{X}\right)$ and $\left(X, D_{X}\right)$ is $\log$ canonical. Then $Z$ is normal and has at most rational singularities. Let $\Delta$ be an ample $\mathbb{Q}$-Cartier $\mathbb{Q}$-divisor on $X$. Then there exists an effective $\mathbb{Q}$ divisor $B_{Z}$ on the variety $Z$ such that

$$
\left.\left(K_{X}+D_{X}+\Delta\right)\right|_{Z} \sim_{\mathbb{Q}} K_{Z}+B_{Z},
$$

and $\left(Z, B_{Z}\right)$ has Kawamata $\log$ terminal singularities. 
Let $\bar{G} \subseteq \operatorname{Aut}(X)$ be a finite subgroup such that $D_{X}$ is $\bar{G}$-invariant. Then $g(Z) \in$ $\mathbb{L} \mathbb{C S}\left(X, D_{X}\right)$ for every $g \in \bar{G}$, and the locus $\operatorname{LCS}\left(X, D_{X}\right)$ is $\bar{G}$-invariant.

If $Z$ is a minimal center in $\mathbb{L} \mathbb{C S}\left(X, D_{X}\right)$ and $\left(X, D_{X}\right)$ is log canonical, then it follows from Lemma 2.6 that

$$
Z \cap g(Z) \neq \varnothing \Longleftrightarrow Z=g(Z)
$$

for every $g \in \bar{G}$.

Lemma 2.8 Suppose that $Z$ is a minimal center in $\mathbb{L} \mathbb{C}\left(X, D_{X}\right)$, the log pair $\left(X, D_{X}\right)$ is $\log$ canonical, and $D_{X}$ is ample. Let $\epsilon$ be an arbitrary rational number such that $\epsilon>1$. Then there exists an effective $\bar{G}$-invariant $\mathbb{Q}$-divisor $D$ on the variety $X$ such that

$$
\mathbb{L} \mathbb{C S}(X, D)=\bigcup_{g \in \bar{G}}\{g(Z)\},
$$

the log pair $(X, D)$ is log canonical, and the equivalence $D \sim_{\mathbb{Q}} \in D_{X}$ holds.

Proof Take $m \in \mathbb{Z}$ such that $m D_{X}$ is a very ample Cartier divisor. Take a general divisor $R$ in the linear system $\left|n m D_{X}\right|$ such that $Z \subset \operatorname{Supp}(R)$ and $R$ is $\bar{G}$-invariant, where $n \gg 0$. Then

$$
\bigcup_{g \in \bar{G}}\{g(Z)\} \subseteq \mathbb{L} \mathbb{C S}\left(X, \lambda D_{X}+\mu R\right) \subseteq \mathbb{L} \mathbb{C} \mathbb{S}\left(X, D_{X}\right)
$$

for some positive rational numbers $\lambda$ and $\mu$ such that $\lambda<1 \leqslant \lambda+\mu n m<\epsilon$. One has $\lambda D_{X}+\mu R \sim_{\mathbb{Q}}(\lambda+\mu n m) D_{X}$.

It follows from the generality of the divisor $R$ that $(X, \mu R)$ is Kawamata log terminal, and

$$
\mathbb{L} \mathbb{C} S\left(X, \lambda D_{X}+\mu R\right)=\bigcup_{g \in \bar{G}} g(Z),
$$

because $\lambda<1$ and $n \gg 0$. Then there is $\theta \in \mathbb{Q}_{>0}$ such that $0<1-\theta \mu \leqslant \lambda<1$ and

$$
\bigcup_{g \in \bar{G}}\{g(Z)\} \subseteq \mathbb{L} \mathbb{C} \mathbb{S}\left(X,(1-\theta \mu) D_{X}+\mu R\right) \subseteq \mathbb{L} \mathbb{C} \mathbb{S}\left(X, \lambda D_{X}+\mu R\right),
$$

but the log pair $\left(X,(1-\theta \mu) D_{X}+\mu R\right)$ is $\log$ canonical at the general point of $Z$.

Note that for a fixed $R$, the number $\theta$ is a function of $\mu$. In the above process, we can choose the number $\mu$ so that $1 \leqslant 1-\theta \mu+\mu n m<\epsilon$ and

$$
\mathbb{L} \mathbb{C S}\left(X,(1-\theta \mu) D_{X}+\mu R\right)=\bigcup_{g \in \bar{G}}\{g(Z)\},
$$


because $Z$ is a minimal center in $\mathbb{L} \mathbb{C S}\left(X, D_{X}\right)$ (see Lemma 2.6). Put

$$
D=(1-\theta \mu) D_{X}+\mu R+\frac{\epsilon-1-\theta \mu+\mu n m}{n m} M,
$$

where $M$ is a general $\bar{G}$-invariant divisor in $|R|$. Then $D$ is the required divisor.

Suppose now that $X=\mathbb{P}^{n}$. In this case we can say much more about the locus $\operatorname{LCS}\left(X, D_{\mathbb{P}^{n}}\right)$ and the set $\mathbb{L} \mathbb{C S}\left(\mathbb{P}^{n}, D_{\mathbb{P}^{n}}\right)$.

Lemma 2.9 Let $H$ be a hyperplane in $\mathbb{P}^{n}$, and let $\mu$ be a nonnegative rational number such that $D_{\mathbb{P}^{n}} \sim_{\mathbb{Q}} \mu H$. Suppose that the locus $\operatorname{LCS}\left(\mathbb{P}^{n}, D_{\mathbb{P}^{n}}\right)$ is an equidimensional subvariety in $\mathbb{P}^{n}$ of codimension $s$. Put

$$
r= \begin{cases}\lceil\mu-s-1\rceil & \text { if } \mu \notin \mathbb{Z}, \\ \lceil\mu-s-1\rceil+1 & \text { if } \mu \in \mathbb{Z} .\end{cases}
$$

Then $r \geqslant 0$ and

$$
\operatorname{deg}\left(\operatorname{LCS}\left(\mathbb{P}^{n}, D_{\mathbb{P}^{n}}\right)\right) \leqslant\left(\begin{array}{c}
s+r \\
r
\end{array}\right)
$$

Proof Put $Y=\operatorname{LCS}\left(\mathbb{P}^{n}, D_{\mathbb{P}^{n}}\right)$. Let $\Pi \subset \mathbb{P}^{n}$ be a general linear subspace of dimension $s$. Put $D=\left.D_{\mathbb{P}^{n}}\right|_{\Pi}$ and $\Lambda=H \cap \Pi$. Then $\operatorname{deg}(Y)=|Y \cap \Pi|$ and $\operatorname{LCS}(\Pi, D)=Y \cap \Pi$ by Remark 2.5. One has $K_{\Pi}+D \sim_{\mathbb{Q}}(\mu-s-1) \Lambda$.

It follows from Theorem 2.3 that there is an exact sequence of cohomology groups

$$
0 \longrightarrow H^{0}\left(\mathcal{O}_{\Pi}(r \Lambda) \otimes \mathcal{I}(\Pi, D)\right) \longrightarrow H^{0}\left(\mathcal{O}_{\Pi}(r \Lambda)\right) \longrightarrow H^{0}\left(\mathcal{O}_{\mathcal{L}(\Pi, D)}\right) \longrightarrow 0
$$

and $\operatorname{Supp}(\mathcal{L}(\Pi, D))=\operatorname{LCS}(\Pi, D)=Y \cap \Pi \neq \varnothing$. Therefore, we see that $r \geqslant 0$ and

$$
\operatorname{deg}(Y)=|Y \cap \Pi| \leqslant h^{0}\left(\mathcal{O}_{\mathcal{L}(\Pi, D)}\right) \leqslant h^{0}\left(\mathcal{O}_{\Pi}(r \Lambda)\right)=h^{0}\left(\mathcal{O}_{\mathbb{P}^{s}}(r)\right)=\left(\begin{array}{c}
s+r \\
r
\end{array}\right),
$$

which completes the proof.

Let $\phi: \mathrm{GL}_{n+1}(\mathbb{C}) \rightarrow \operatorname{Aut}\left(\mathbb{P}^{n}\right) \cong \mathrm{PGL}_{n+1}(\mathbb{C})$ be the natural projection, and let $G$ be a finite subgroup in $\mathrm{GL}_{n+1}(\mathbb{C})$ such that $\bar{G}=\phi(G)$.

Remark 2.10 If $G$ does not have semi-invariants of degree at most $k$, then every $\bar{G}$-orbits in $\mathbb{P}^{n}$ contains at least $k+1$ points, because every $\bar{G}$-orbit consisting of $s$ points defines a $\bar{G}$-invariant hypersurface in $\mathbb{P}^{n}$ that is a union of $s$ hyperplanes. 
Lemma 2.11 Let $H$ be a hyperplane in $\mathbb{P}^{n}$, and let $\mu$ be a nonnegative rational number such that $D_{\mathbb{P}^{n}} \sim_{\mathbb{Q}} \mu H$. Suppose that $G$ does not have semi-invariants of degree at most $\lfloor\mu\rfloor$. Then $\mathbb{L} \mathbb{C} S\left(\mathbb{P}^{n}, D_{\mathbb{P}^{n}}\right)$ does not contain subvarieties in $\mathbb{P}^{n}$ of codimension 1. If in addition $\lfloor\mu\rfloor \leqslant n+1$ and the $\log$ pair $\left(\mathbb{P}^{n}, D_{\mathbb{P}^{n}}\right)$ is $\log$ canonical, then $\mathbb{L} \mathbb{C S}\left(\mathbb{P}^{n}, D_{\mathbb{P}^{n}}\right)$ does not contain points.

Proof Suppose that $\mathbb{L} \mathbb{C S}\left(\mathbb{P}^{n}, D_{\mathbb{P}^{n}}\right)$ contains an irreducible subvariety $Y \subset \mathbb{P}^{n}$ of codimension 1 . Let $R$ be the $\bar{G}$-orbit of the subvariety $Y$. Then

$$
D_{\mathbb{P}^{n}}=a R+\Delta
$$

for some rational number $a \geqslant 1$ and some effective $\mathbb{Q}$-divisor $\Delta$ on $\mathbb{P}^{n}$. Since $D_{\mathbb{P}^{n}} \sim \mathbb{Q} \mu H$, we see that $R$ is a hypersurface in $\mathbb{P}^{n}$ of degree at most $\lfloor\mu / a\rfloor \leqslant\lfloor\mu\rfloor$, which is impossible, because $G$ does not have semi-invariants of degree at most $\lfloor\mu\rfloor$. We see that $\mathbb{L} \mathbb{C S}\left(\mathbb{P}^{n}, D_{\mathbb{P}^{n}}\right)$ does not contain subvarieties in $\mathbb{P}^{n}$ of codimension 1 . Let us show that $\mathbb{L} \mathbb{C}\left(\mathbb{P}^{n}, D_{\mathbb{P}^{n}}\right)$ does not contain points provided that $\lfloor\mu\rfloor \leqslant n+1$ and the log pair $\left(\mathbb{P}^{n}, D_{\mathbb{P}^{n}}\right)$ is log canonical.

Suppose that $\lfloor\mu\rfloor \leqslant n+1$, the log pair $\left(\mathbb{P}^{n}, D_{\mathbb{P}^{n}}\right)$ is log canonical, and $\mathbb{L} \mathbb{C}\left(\mathbb{P}^{n}, D_{\mathbb{P}}\right)$ contains a point $P \in \mathbb{P}^{n}$. Let us show that these assumptions lead to a contradiction.

Let $\Sigma$ be the $\bar{G}$-orbit of the point $P$, and let $\epsilon$ be a rational number such that $\epsilon>1$ and $\lfloor\epsilon \mu\rfloor \leqslant n+1$. Then it follows from Lemma 2.8 that there is an effective $\bar{G}$-invariant $\mathbb{Q}$-divisor $D$ on $\mathbb{P}^{n}$ such that $D \sim_{\mathbb{Q}} \epsilon \mu H$, the log pair $\left(\mathbb{P}^{n}, D\right)$ is log canonical and $\Sigma=\operatorname{LCS}\left(\mathbb{P}^{n}, D\right)$.

Since $\lfloor\epsilon \mu\rfloor \leqslant n+1$, it follows from Theorem 2.3 that

$$
H^{0}\left(\mathcal{O}_{\mathbb{P}^{n}}(1) \otimes \mathcal{I}\left(\mathbb{P}^{n}, D\right)\right)=0,
$$

because $K_{\mathbb{P}^{n}}+D \sim_{\mathbb{Q}}(\epsilon \mu-n-1) H$ and $\epsilon \mu-n-1<1$. Therefore, it follows from the exact sequence of cohomology groups

$$
0 \longrightarrow H^{0}\left(\mathcal{O}_{\mathbb{P}^{n}}(1) \otimes \mathcal{I}\left(\mathbb{P}^{n}, D\right)\right) \longrightarrow H^{0}\left(\mathcal{O}_{\mathbb{P}^{n}}(1)\right) \longrightarrow H^{0}\left(\mathcal{O}_{\Sigma}\right) \longrightarrow 0
$$

that $|\Sigma| \leqslant n+1$, which is impossible because $G$ does not have semi-invariants of degree at most $\lfloor\mu\rfloor \leqslant n+1$.

Remark 2.12 If $G$ is conjugate to a subgroup in $\mathrm{GL}_{n+1}(\mathbb{R})$, then the subgroup $G$ has an invariant of degree 2 , which implies that $\operatorname{lct}\left(\mathbb{P}^{n}, \bar{G}\right) \leqslant 2 /(n+1)$.

Remark 2.13 If $Z$ is a $\bar{G}$-invariant, then there is a homomorphism $\xi: \bar{G} \rightarrow \operatorname{Aut}(Z)$ that must be a monomorphism provided that $Z$ is not contained in a linear subspace of $\mathbb{P}^{n}$, because eigenvectors that correspond to a fixed eigenvalue of any matrix in $\mathrm{GL}_{n+1}(\mathbb{C})$ form a vector subspace in $\mathbb{C}^{n+1}$. 
Theorem 2.14 Let $C$ be a smooth irreducible curve of genus $g \geqslant 2$. Then $|\operatorname{Aut}(C)| \leqslant$ $84(g-1)$.

Proof The required inequality is the famous Hurwitz bound (see Breuer [6, Theorem 3.17]).

\section{Exceptionality criterion}

Let $X$ be a variety, let $B_{X}$ be an effective $\mathbb{Q}$-divisor on $X$ such that the log pair $\left(X, B_{X}\right)$ has at most Kawamata log terminal singularities, and the divisor $-\left(K_{X}+B_{X}\right)$ is ample. Recall that $\left(X, B_{X}\right)$ is usually called a log Fano variety. Let $\bar{G} \subset \operatorname{Aut}(X)$ be a finite subgroup such that the divisor $B_{X}$ is $\bar{G}$-invariant.

Definition 3.1 The global $\bar{G}$-invariant log canonical threshold of the log Fano variety $\left(X, B_{X}\right)$ is a real number $\operatorname{lct}\left(X, B_{X}, \bar{G}\right)$ that can be defined as

$$
\inf \left\{\begin{array}{l|l}
\mathrm{c}\left(X, B_{X}, D_{X}\right) \in \mathbb{Q} & \begin{array}{l}
D_{X} \text { is a } \bar{G} \text {-invariant } \mathbb{Q} \text {-Cartier effective } \mathbb{Q} \text {-divisor } \\
\text { on the variety } X \text { such that } D_{X} \sim_{\mathbb{Q}}-\left(K_{X}+B_{X}\right)
\end{array}
\end{array}\right\} .
$$

For simplicity, we put $\operatorname{lct}\left(X, B_{X}, \bar{G}\right)=\operatorname{lct}(X, \bar{G})$ if $B_{X}=0$. Similarly, we put $\operatorname{lct}\left(X, B_{X}, \bar{G}\right)=\operatorname{lct}\left(X, B_{X}\right)$ if $\bar{G}$ is trivial. Finally, we put $\operatorname{lct}\left(X, B_{X}, \bar{G}\right)=\operatorname{lct}(X)$ if $B_{X}=0$ and $\bar{G}$ is trivial. Then it follows from [11, Theorem A.3] that $\operatorname{lct}(X, \bar{G})=$ $\alpha_{\bar{G}}(X)$ if $X$ is smooth and $B_{X}=0$ (see Definition 1.2).

Remark 3.2 Suppose that $B_{X}=0$. Put $V=X / \bar{G}$. Let $\theta: X \rightarrow V$ be the quotient map. Then

$$
K_{X} \sim \mathbb{Q} \theta^{*}\left(K_{V}+R_{V}\right),
$$

where $R_{V}$ is a ramification $\mathbb{Q}$-divisor of the morphism $\theta$. Note that $-\left(K_{V}+R_{V}\right)$ is an ample $\mathbb{Q}$-Cartier divisor, and $\left(V, R_{V}\right)$ is Kawamata log terminal by [23, Proposition 3.16]. Moreover, it follows from [23, Proposition 3.16] that $\operatorname{lct}(X, \bar{G})=$ $\operatorname{lct}\left(V, R_{V}\right)$.

Example 3.3 Suppose that $X \cong \mathbb{P}^{1}$. Then $B_{X}=\sum_{i=1}^{n} a_{i} P_{i}$, where $P_{i}$ is a point, and $a_{i} \in \mathbb{Q}$ such that $0 \leqslant a_{i}<1$. We may assume that $a_{0} \leqslant \ldots \leqslant a_{n}$. Then

$$
\operatorname{lct}\left(X, B_{X}\right)=\frac{1-a_{n}}{2-\sum_{i=1}^{n} a_{i}}
$$

where $\sum_{i=1}^{n} a_{i}<2$, because the divisor $-\left(K_{X}+B_{X}\right)$ is ample. Moreover, it follows from Remark 3.2 that $\operatorname{lct}(X, \bar{G})=2 / \lambda$, where $\lambda$ is the length of a $\bar{G}$-orbit of the smallest length (cf Theorem 4.1). 
Lemma 3.4 The global $\log$ canonical threshold $\operatorname{lct}\left(X, B_{X}, \bar{G}\right)$ is equal to $\inf \left\{\begin{array}{l|l}\mathrm{c}\left(X, B_{X}, \sum_{i=1}^{r} a_{i} \mathcal{D}_{i}\right) & \begin{array}{l}\mathcal{D}_{i} \text { is a linear system and } a_{i} \in \mathbb{Q} \geqslant 0 \\ \text { for every } i \in\{1, \ldots, r\}, \sum_{i=1}^{r} a_{i} \mathcal{D}_{i} \text { is } \bar{G} \text {-invariant, } \\ \text { and } \sum_{i=1}^{r} a_{i} \mathcal{D}_{i} \sim_{\mathbb{Q}}-\left(K_{X}+B_{X}\right)\end{array}\end{array}\right\}$.

Proof The required assertion follows from Definition 3.1 and [23, Theorem 4.8].

In general, it is unknown whether $\operatorname{lct}\left(X, B_{X}, \bar{G}\right)$ is a rational number or not (cf [43, Question 1]). Of course, we expect that $\operatorname{lct}\left(X, B_{X}, \bar{G}\right)$ is rational. Moreover, we expect the following to be true.

Conjecture 3.5 There is an effective $\bar{G}$-invariant $\mathbb{Q}$-divisor $D_{X}$ on $X$ such that $\operatorname{lct}\left(X, B_{X}, \bar{G}\right)=\mathrm{c}\left(X, B_{X}, D_{X}\right) \in \mathbb{Q}$ and $D_{X} \sim_{\mathbb{Q}}-\left(K_{X}+B_{X}\right)$.

Let $(V \ni O)$ be a germ of a Kawamata log terminal singularity, and let $\pi: W \rightarrow V$ be a birational morphism such that the exceptional locus of $\pi$ consists of one irreducible divisor $E \subset W$ such that $O \in \pi(E)$, the $\log$ pair $(W, E)$ has purely log terminal singularities (see [23, Definition 3.5]), and $-E$ is a $\pi$-ample $\mathbb{Q}$-Cartier divisor.

Theorem 3.6 The birational morphism $\pi: W \rightarrow V$ does exist.

Proof Modulo the Log Minimal Model Program in dimension $\operatorname{dim}(V)$, the existence of the morphism $\pi$ follows from [31, Proposition 2.9] in the case when $V$ has $\mathbb{Q}$ factorial singularities. It follows from [24, Theorem 1.5] that the $\mathbb{Q}$-factoriality condition in [31, Proposition 2.9] can be removed. Moreover, the proofs of [31, Proposition 2.9] and [24, Theorem 1.5] only need the Log Minimal Model Program for log pairs with big boundaries, which is proved now in [2].

We say that $\pi: W \rightarrow V$ is a plt blow up of the singularity $(V \ni O)$.

Definition 3.7 [31, Definition 4.1] We say that $(V \ni O)$ is weakly-exceptional if it has unique plt blow up.

Weakly-exceptional Kawamata log terminal singularities do exist (see [24, Example 2.2]).

Lemma 3.8 [24, Corollary 1.7] If $(V \ni O)$ is weakly-exceptional, then $\pi(E)=O$. 
Let $R_{1}, \ldots, R_{S}$ be irreducible components of $\operatorname{Sing}(W) \operatorname{such}$ that $\operatorname{dim}\left(R_{i}\right)=\operatorname{dim}(W)-2$ and $R_{i} \subset E$ for every $i \in\{1, \ldots, s\}$. Put

$$
\operatorname{Diff}_{E}(0)=\sum_{i=1}^{s} \frac{m_{i}-1}{m_{i}} R_{i},
$$

where $m_{i}$ is the smallest positive integer such that $m_{i} E$ is Cartier at a general point of $R_{i}$.

Lemma 3.9 [23, Theorem 7.5] The variety $E$ is normal, and $\left(E, \operatorname{Diff}_{E}(0)\right)$ is Kawamata log terminal.

Therefore, if $\pi(E)=O$, then the $\log$ pair $\left(E\right.$, Diff $\left._{E}(0)\right)$ is a log Fano variety, because $-E$ is $\pi$-ample.

Theorem 3.10 [24, Theorem 2.1] The singularity $(V \ni O)$ is weakly-exceptional if and only if $\pi(E)=O$ and $\operatorname{lct}\left(E, \operatorname{Diff}_{E}(0)\right) \geqslant 1$.

Theorem 3.11 [31, Theorem 4.9] The singularity $(V \ni O)$ is exceptional if and only if $\pi(E)=O$ and $\mathrm{c}\left(E, \operatorname{Diff}_{E}(0), D_{E}\right)>1$ for every effective $\mathbb{Q}$-divisor $D_{E}$ on the variety $E$ such that $D_{E} \sim \mathbb{Q}-\left(K_{E}+\operatorname{Diff}_{E}(0)\right)$.

In particular, we see that if the assertion of Conjecture 3.5 is true, then $(V \ni O)$ is exceptional if and only if $\pi(E)=O$ and $\operatorname{lct}\left(E, \operatorname{Diff}_{E}(0)\right)>1$ holds.

Corollary 3.12 If $(V \ni O)$ is exceptional, then $(V \ni O)$ is weakly-exceptional.

It should be pointed out that Theorem 3.11 is an applicable criterion. For instance, it can be used to construct exceptional singularities of any dimension.

Example 3.13 Suppose that $(V \ni O)$ is a Brieskorn-Pham hypersurface singularity

$$
\sum_{i=0}^{n} x_{i}^{a_{i}}=0 \subset \mathbb{C}^{n+1} \cong \operatorname{Spec}\left(\mathbb{C}\left[x_{0}, x_{1}, \ldots, x_{n}\right]\right),
$$

where $n \geqslant 3$ and $2 \leqslant a_{0}<a_{1}<\cdots<a_{n}$. Arguing as in the proof of [4, Theorem 34], we see that it follows from Theorem 3.11 that the singularity $(V \ni O)$ is exceptional if

$$
1<\sum_{i=0}^{n} \frac{1}{a_{i}}<1+\min \left\{\frac{1}{a_{0}}, \frac{1}{a_{1}}, \ldots, \frac{1}{a_{n}}\right\}
$$


and $a_{0}, a_{1}, \ldots, a_{n}$ are pairwise coprime. This is satisfied if $a_{0}, a_{1}, \ldots, a_{n}$ are primes and

$$
\frac{1}{a_{0}}+\frac{1}{a_{1}}+\cdots+\frac{1}{a_{n-1}}<1<\frac{1}{a_{0}}+\frac{1}{a_{1}}+\cdots+\frac{1}{a_{n-1}}+\frac{1}{a_{n}} .
$$

We use induction to construct the $(n+1)$-tuple $\left(a_{0}, a_{1}, \ldots, a_{n}\right)$ such that $a_{0}, a_{1}, \ldots, a_{n}$ are prime integers, and the $(n+1)$-tuple $\left(a_{0}, a_{1}, \ldots, a_{n}\right)$ satisfies the inequality (3.14). If $n=3$, then the four-tuple $\left(a_{0}, a_{1}, a_{2}, a_{3}\right)=(2,3,7,41)$ satisfies the inequality (3.14). Suppose that $n \geqslant 4$, and there are prime numbers $2 \leqslant c_{0}<c_{1}<c_{2}<\cdots<c_{n-1}$ such that

$$
\frac{1}{c_{0}}+\frac{1}{c_{1}}+\cdots+\frac{1}{c_{n-2}}<1<\frac{1}{c_{0}}+\frac{1}{c_{1}}+\cdots+\frac{1}{c_{n-2}}+\frac{1}{c_{n-1}}
$$

and assume that $c_{n-1}>8$ is the largest prime with these properties (for the fixed numbers $\left.c_{0}, \ldots, c_{n-2}\right)$. It follows from $c_{n-1}>8$ that there are prime numbers $p_{1}, p_{2}$ and $p_{3}$ such that $c_{n-1}<p_{1}<p_{2}<p_{3}<2 c_{n-1}$ (see [34, page 209, (18)]). Put $\left(a_{0}, a_{1}, \ldots, a_{n}\right)=\left(c_{0}, \ldots, c_{n-2}, p_{2}, p_{3}\right)$. Then

$$
\sum_{i=0}^{n-2} \frac{1}{a_{i}}+\frac{1}{p_{2}}<\sum_{i=0}^{n-2} \frac{1}{a_{i}}+\frac{1}{p_{1}} \leqslant 1<\sum_{i=0}^{n-2} \frac{1}{c_{i}}+\frac{1}{2 c_{n-1}}+\frac{1}{2 c_{n-1}}<\sum_{i=0}^{n-2} \frac{1}{a_{i}}+\frac{1}{p_{2}}+\frac{1}{p_{3}}
$$

by the maximality assumption imposed on $c_{n-1}$. So the $(n+1)$-tuple $\left(a_{0}, a_{1}, \ldots, a_{n}\right)$ satisfies the inequality (3.14), which completes the construction ${ }^{1}$.

Suppose, in addition, that $(V \ni O)$ is a quotient singularity $\mathbb{C}^{n+1} / G$, where $n \geqslant 1$ and $G$ is a finite subgroup in $\mathrm{GL}_{n+1}(\mathbb{C})$. Put $\bar{G}=\phi(G)$, where $\phi: \mathrm{GL}_{n+1}(\mathbb{C}) \rightarrow$ $\operatorname{Aut}\left(\mathbb{P}^{n}\right) \cong \operatorname{PGL}_{n+1}(\mathbb{C})$ is the natural projection.

Remark 3.15 Let $\eta: \mathbb{C}^{n+1} \rightarrow V$ be the quotient map. Then there is a commutative diagram

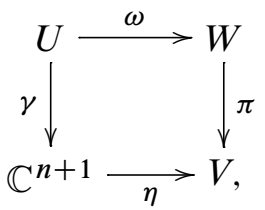

where $\gamma$ is the blow up of $O$, the morphism $\omega$ is the quotient map that is induced by the lifted action of $G$ on the variety $U$, and $\pi$ is a birational morphism. Moreover, $\pi$ is a plt blow up of the singularity $\mathbb{C}^{n+1} / G$.

1 Alternatively, one can use the Sylvester sequence to construct $\left(a_{0}, \ldots, a_{n}\right)$ explicitly (suggested by S. Galkin). 
Thus, to prove the existence of a plt blow up of the quotient singularity $\mathbb{C}^{n+1} / G$ we do not need to use Theorem 3.6.

Theorem 3.16 Suppose that the group $G \subset \mathrm{GL}_{n+1}(\mathbb{C})$ does not contain reflections. Then the singularity $\mathbb{C}^{n+1} / G$ is weakly-exceptional if and only if $\operatorname{lct}\left(\mathbb{P}^{n}, \bar{G}\right) \geqslant 1$.

Proof Let us use the notation and assumptions of Remark 3.15. Let $F$ be the exceptional divisor of the blow up $\gamma$. Put $E=\omega(F)$. Then $F \cong \mathbb{P}^{n}$ and $E \cong \mathbb{P}^{n} / \bar{G}$. Since the group $G$ does not contain reflections, it follows from Remark 3.2 that $\operatorname{lct}\left(\mathbb{P}^{n}, \bar{G}\right)=\operatorname{lct}\left(E, \operatorname{Diff}_{E}(0)\right)$, which implies that the singularity $\mathbb{C}^{n+1} / G$ is weaklyexceptional if and only if $\operatorname{lct}\left(\mathbb{P}^{n}, \bar{G}\right) \geqslant 1$ by Theorem 3.11 .

Theorem 3.17 Suppose that the group $G \subset \mathrm{GL}_{n+1}(\mathbb{C})$ does not contain reflections. Then the singularity $\mathbb{C}^{n+1} / G$ is exceptional if and only if for any $\bar{G}$-invariant effective $\mathbb{Q}$-divisor $D$ on $\mathbb{P}^{n}$ such that $D \sim_{\mathbb{Q}}-K_{\mathbb{P}^{n}}$ the $\log$ pair $\left(\mathbb{P}^{n}, D\right)$ is Kawamata $\log$ terminal.

Proof Arguing as in the proof of Theorem 3.16 and using Theorem 3.11 together with [23, Proposition 3.16], we obtain the required assertion.

Recall that the subgroup $G \subset \mathrm{GL}_{n+1}(\mathbb{C})$ is said to be transitive if the corresponding $(n+1)$-dimensional representation is irreducible (see [3]). Note that $G$ is transitive if it is primitive. As an easy application of Theorems 3.17 and 3.16 in conjunction with Lemma 3.4 one can establish the relation between the primitivity of the group $G$ (transitivity, respectively) and the exceptionality of the singularity $\mathbb{C}^{n+1} / G$ (weakexceptionality, respectively).

Theorem 3.18 Suppose that the group $G \subset \mathrm{GL}_{n+1}(\mathbb{C})$ is not primitive (not transitive, respectively). Then there exists a $\bar{G}$-invariant effective $\mathbb{Q}$-divisor $D$ on $\mathbb{P}^{n}$ such that $D \sim \mathbb{Q}-K_{\mathbb{P}^{n}}$ and the pair $\left(\mathbb{P}^{n}, D\right)$ is not Kawamata log terminal (not log canonical, respectively).

Proof We will only prove that if the group $G$ is not primitive, then there exists a $\bar{G}-$ invariant effective $\mathbb{Q}$-divisor $D$ on $\mathbb{P}^{n}$ such that $D \sim \mathbb{Q}-K_{\mathbb{P}^{n}}$ and the pair $\left(\mathbb{P}^{n}, D\right)$ is not Kawamata log terminal, since the remaining assertion can be proved similarly.

Suppose that $G$ is not primitive. Then there is a nontrivial decomposition

$$
\operatorname{Spec}\left(\mathbb{C}\left[x_{0}, x_{1}, \ldots, x_{n}\right]\right) \cong \mathbb{C}^{n+1}=\bigoplus_{i=1}^{r} V_{i}
$$


such that $g\left(V_{i}\right)=V_{j}$ for all $g \in G$. We may assume that $\operatorname{dim}\left(V_{1}\right) \leqslant \ldots \leqslant \operatorname{dim}\left(V_{r}\right)$. Put $d=\operatorname{dim}\left(V_{1}\right)$. Then $d \leqslant\lfloor(n+1) / 2\rfloor$. We may assume that $V_{1} \subset \mathbb{C}^{n+1}$ is given by $x_{d}=x_{d+1}=x_{d+2}=\cdots=x_{n}=0$. Let $\mathcal{M}_{1}$ be a linear system on $\mathbb{P}^{n}$ that consists of hyperplanes that are given by

$$
\sum_{i=0}^{d-1} \lambda_{i} x_{i}=0 \subset \mathbb{P}^{n} \cong \operatorname{Proj}\left(\mathbb{C}\left[x_{0}, x_{1}, \ldots, x_{n}\right]\right),
$$

where $\lambda_{i} \in \mathbb{C}$. Let $\mathcal{M}_{1}, \ldots, \mathcal{M}_{s}$ be the $\bar{G}$-orbit of the linear system $\mathcal{M}_{1}$. Then

$$
\frac{n+1}{s}\left(\sum_{i=1}^{s} \mathcal{M}_{i}\right) \sim_{\mathbb{Q}}-K_{\mathbb{P}^{n}},
$$

where $s \leqslant\lfloor(n+1) / d\rfloor$. Let $\Lambda \subset \mathbb{P}^{n}$ be a linear subspace that is given by the equations $x_{0}=\ldots=x_{d}=0$. Then

$$
\frac{n+1}{s} \operatorname{mult}_{\Lambda}\left(\sum_{i=1}^{s} \mathcal{M}_{i}\right) \geqslant \frac{n+1}{s} \operatorname{mult}_{\Lambda}\left(\mathcal{M}_{1}\right)=\frac{n+1}{s} \geqslant d=n-\operatorname{dim}(\Lambda),
$$

which implies the desired assertion by Lemma 3.4.

Corollary 3.19 Suppose that the group $G \subset \mathrm{GL}_{n+1}(\mathbb{C})$ is not primitive (not transitive, respectively). Then $\operatorname{lct}\left(\mathbb{P}^{n}, \bar{G}\right) \leqslant 1\left(\operatorname{lct}\left(\mathbb{P}^{n}, \bar{G}\right)<1\right.$, respectively).

Applying Theorems 3.16, 3.17 and 3.18, we obtain the following.

Corollary 3.20 [32, Proposition 2.1] Suppose that the group $G \subset \mathrm{GL}_{n+1}(\mathbb{C})$ does not contain reflections. Then the group $G$ is primitive (transitive, respectively) provided that the singularity $\mathbb{C}^{n+1} / G$ is exceptional (weakly-exceptional, respectively).

Let us show how to apply Theorems 3.16 and 3.17 (cf [9, Example 1.9]).

Theorem 3.21 Suppose that $G \subset \mathrm{GL}_{3}(\mathbb{C})$. Then $\operatorname{lct}\left(\mathbb{P}^{2}, \bar{G}\right) \geqslant 4 / 3$ if and only if $G$ does not have semi-invariants of degree at most 3 .

Proof Suppose that the subgroup $G$ does not have semi-invariants of degree at most 3 . To complete the proof we must show that $\operatorname{lct}\left(\mathbb{P}^{2}, \bar{G}\right) \geqslant 4 / 3$, because the remaining implication is obvious.

Suppose that the strict inequality $\operatorname{lct}\left(\mathbb{P}^{2}, \bar{G}\right)<4 / 3$ holds. Then there exist a positive rational number $\lambda<4 / 3$ and an effective $\bar{G}$-invariant $\mathbb{Q}$-divisor $D$ on $\mathbb{P}^{2}$ such that $D \sim \mathbb{Q}-K_{\mathbb{P}^{2}}$, and the $\log$ pair $\left(\mathbb{P}^{2}, \lambda D\right)$ is strictly log canonical. Applying Lemma 2.11, we obtain a contradiction. 
Using Theorems 3.17 and 3.21, we obtain the following.

Corollary 3.22 Suppose that the group $G \subset \mathrm{GL}_{3}(\mathbb{C})$ does not contain reflections. Then the following are equivalent:

- The singularity $\mathbb{C}^{3} / G$ is exceptional.

- The subgroup $G$ does not have semi-invariants of degree at most 3 .

- $\quad$ The inequality $\operatorname{lct}\left(\mathbb{P}^{2}, \bar{G}\right) \geqslant 4 / 3$ holds.

Arguing as in the proof of Theorem 3.21, we easily obtain a similar assertion that can be used for the classification of three-dimensional weakly exceptional quotient singularities (see [36]).

Theorem 3.23 Suppose that $G \subset \mathrm{GL}_{3}(\mathbb{C})$. Then $\operatorname{lct}\left(\mathbb{P}^{2}, \bar{G}\right) \geqslant 1$ if and only if $G$ does not have semi-invariants of degree at most 2.

Proof The proof is left to the reader.

Suppose that $n+1=2 l$ for some integer $l \geqslant 2$. Let $G_{1} \subset \mathrm{SL}_{2}(\mathbb{C})$ and $G_{2} \subset \mathrm{SL}_{l}(\mathbb{C})$ be finite subgroups, let $\mathbb{M}$ be the vector space of $(2 \times l)$-matrices with entries in $\mathbb{C}$. For every $\left(g_{1}, g_{2}\right) \in G_{1} \times G_{2}$ and every $M \in \mathbb{M}$, put

$$
\left(g_{1}, g_{2}\right)(M)=g_{1} M g_{2}^{-1} \in \mathbb{M} \cong \mathbb{C}^{2 l},
$$

which induces a homomorphism $\varphi: G_{1} \times G_{2} \rightarrow \operatorname{SL}_{2 l}(\mathbb{C})$. Note that $|\operatorname{ker}(\varphi)| \leqslant 2$ if $n$ is even, and $\varphi$ is a monomorphism if $n$ is odd.

Lemma 3.24 Suppose that $G=\varphi\left(G_{1} \times G_{2}\right)$. Then $\operatorname{lct}\left(\mathbb{P}^{n}, \bar{G}\right)<1$.

Proof Put $s=l-1$. Let $\psi: \mathbb{P}^{1} \times \mathbb{P}^{s} \rightarrow \mathbb{P}^{n}$ be the Segre embedding. Put $Y=$ $\psi\left(\mathbb{P}^{1} \times \mathbb{P}^{s}\right)$ and let $\mathcal{Q}$ be the linear system consisting of all quadric hypersurfaces in $\mathbb{P}^{n}$ that pass through the subvariety $Y$. Then $\mathcal{Q}$ is a nonempty $\bar{G}$-invariant linear system. The log pair $\left(\mathbb{P}^{n}, l \mathcal{Q}\right)$ is not log-canonical along $Y$, which implies that $\operatorname{lct}\left(\mathbb{P}^{n}, \bar{G}\right)<1$ by Lemma 3.4.

As an application of Lemma 3.24 one obtains nonexceptionality of some quotient singularities.

Example 3.25 (cf Theorem 1.22) Suppose that $G=\varphi\left(G_{1} \times G_{2}\right)$ and $l=3$. Then the singularity $\mathbb{C}^{6} / G$ is not exceptional by Theorem 1.17 and Lemma 3.24. On the other hand, if $G_{1} \cong 2$. $_{5}$ and $G_{2} \cong 3$. $\mathrm{A}_{6}$, then $G$ has no semi-invariants of degree at most 6 which can be shown by direct computation. 
Suppose that $l=2$. The transposition of matrices in $\mathbb{M}$ induces an involution $\iota \in$ $\mathrm{SL}_{4}(\mathbb{C})$.

Lemma 3.26 If $G$ is generated by $\varphi\left(G_{1} \times G_{2}\right)$ and $\iota$, then $\operatorname{lct}\left(\mathbb{P}^{3}, \bar{G}\right)<1$.

Proof See the proof of Lemma 3.24.

\section{Four-dimensional case}

Shokurov [38] and Prokhorov and Markushevich [27] obtained an explicit classification of exceptional quotient singularities of dimension 2 and 3. Namely, for Gorenstein quotient singularities they prove the following.

Theorem 4.1 [38, Example 5.2.3] Let $G$ be the finite subgroup in $\mathrm{SL}_{2}(\mathbb{C})$. Then the singularity $\mathbb{C}^{2} / G$ is exceptional if and only if $G$ is a binary central extension of one of the following groups: $\mathrm{A}_{4}, \mathrm{~S}_{4}$ or $\mathrm{A}_{5}$.

Theorem 4.2 [27, Theorem 3.13] Let $G$ be a finite subgroup in $\operatorname{SL}_{3}(\mathbb{C})$. Then the singularity $\mathbb{C}^{3} / G$ is exceptional if and only if $G$ is one of the following subgroups:

- a central extension of $\operatorname{PSL}_{2}\left(\mathbb{F}_{7}\right)$, which is isomorphic to either $\operatorname{PSL}_{2}\left(\mathbb{F}_{7}\right)$ or $\mathbb{Z}_{3} \times \operatorname{PSL}_{2}\left(\mathbb{F}_{7}\right)$,

- a nontrivial central extension 3. $\mathrm{A}_{6}$ of the alternating group $\mathrm{A}_{6}$ by $\mathbb{Z}_{3}$,

- the Hessian group, which can be characterized by the exact sequence

$$
1 \longrightarrow \mathbb{H}\left(3, \mathbb{F}_{3}\right) \longrightarrow G \longrightarrow \mathrm{S}_{4} \longrightarrow 1
$$

where $\mathbb{H}\left(3, \mathbb{F}_{3}\right)$ is the Heisenberg group consisting of all unipotent $(3 \times 3)-$ matrices with entries in $\mathbb{F}_{3}$,

- the normal subgroup of the Hessian group of index 3 that contains $\mathbb{H}\left(3, \mathbb{F}_{3}\right)$.

The purpose of this section is to present an analogous classification for exceptional singularities of dimension 4 (see Theorem 4.13), and prove some relevant results.

Let $\bar{G}$ be a finite subgroup in $\operatorname{Aut}\left(\mathbb{P}^{3}\right)$, and let $\phi: \mathrm{GL}_{4}(\mathbb{C}) \rightarrow \operatorname{Aut}\left(\mathbb{P}^{3}\right)$ be the natural projection. Then there is a finite subgroup in $\mathrm{SL}_{4}(\mathbb{C})$ such that $\phi(G)=\bar{G}$. Moreover, if $G$ is primitive, then it follows from $[3 ; 14]$ that one may assume that $Z(G) \subseteq[G, G]$, where $Z(G)$ and $[G, G]$ are the center and the commutator of the group $G$, respectively. As a warming-up we start with a result that can be applied to a classification of four-dimensional weakly exceptional quotient singularities (see [36]). 
Theorem 4.3 The inequality $\operatorname{lct}\left(\mathbb{P}^{3}, \bar{G}\right) \geqslant 1$ holds if and only if the following three conditions are satisfied: the group $G$ is transitive, the group $G$ does not have semiinvariants of degree at most 3 , and ${ }^{2}$ there is no $\bar{G}$-invariant smooth rational cubic curve in $\mathbb{P}^{3}$.

Proof Let us prove the $\Rightarrow$ part. If $G$ has a semi-invariant of degree at most 3 , then $\operatorname{lct}\left(\mathbb{P}^{3}, \bar{G}\right) \leqslant 3 / 4$ by Definition 3.1. If $G$ is not transitive, then $\operatorname{lct}\left(\mathbb{P}^{3}, \bar{G}\right)<1$ by Corollary 3.19 .

Suppose that there is a $\bar{G}$-invariant smooth rational cubic curve $C \subset \mathbb{P}^{3}$. Let $R \subset \mathbb{P}^{3}$ be the surface that is swept out by lines that are tangent to $C$. Then $\mathrm{c}\left(\mathbb{P}^{3}, R\right)=5 / 6$ the surface $R$ is $\bar{G}$-invariant, and $\operatorname{deg}(R)=4$. Hence, we see that $\operatorname{lct}\left(\mathbb{P}^{3}, \bar{G}\right) \leqslant 5 / 6$.

Let us prove the $\Leftarrow$ part. Suppose that $G$ is transitive, the subgroup $G$ has no semiinvariants of degree at most 3 , there is no $\bar{G}$-invariant smooth rational cubic curve in $\mathbb{P}^{3}$, but $\operatorname{lct}\left(\mathbb{P}^{3}, \bar{G}\right)<1$.

There is an effective $\bar{G}$-invariant $\mathbb{Q}$-divisor $D$ on $\mathbb{P}^{3}$ such that $D \sim_{\mathbb{Q}}-K_{\mathbb{P}^{3}}$ and a positive rational number $\lambda<1$ such that $\left(\mathbb{P}^{3}, \lambda D\right)$ is strictly $\log$ canonical. Let $S$ be an irreducible subvariety of $\mathbb{P}^{3}$ that is a minimal center in $\mathbb{L} \mathbb{C}\left(\mathbb{P}^{3}, \lambda D\right)$. By Lemma 2.8, we may assume that

$$
\mathbb{L} \mathbb{C S}\left(\mathbb{P}^{3}, \lambda D\right)=\bigcup_{g \in \bar{G}}\{g(S)\},
$$

where $\operatorname{dim}(S) \neq 2$, because $G$ has no semi-invariants of degree at most 3 .

The locus $\operatorname{LCS}\left(\mathbb{P}^{3}, \lambda D\right)$ is connected by Corollary 2.4. Then $S$ is $\bar{G}$-invariant by Lemma 2.6. Since the group $G$ is transitive, we see that $S$ is not a point. We see that $S$ is a curve. Then $\operatorname{deg}(S) \leqslant 3$ by Lemma 2.9 , and $S$ is not contained in a plane, because $G$ is transitive. Hence $S$ is a smooth rational cubic curve.

Combining Remark 2.13, Theorem 4.3 and the classification of finite subgroups in $\mathrm{PGL}_{2}(\mathbb{C})$, we easily obtain the following result (cf Theorem 3.23).

Corollary 4.4 The inequality $\operatorname{lct}\left(\mathbb{P}^{3}, \bar{G}\right) \geqslant 1$ holds if the following three conditions are satisfied: the group $G$ is transitive, the group $G$ does not have semi-invariants of degree at most 3 , and the group $\bar{G}$ is not isomorphic to the alternating group $\mathrm{A}_{5}$.

${ }^{2}$ One can show that the third condition of Theorem 4.3 is not redundant. Namely, if $G \subset \mathrm{SL}_{4}(\mathbb{C})$ is a primitive group isomorphic to $2 . \mathrm{A}_{5}$, then $G$ has no semi-invariants of degree at most 3 , but there is a $\bar{G}$-invariant twisted cubic in $\mathbb{P}^{3}$. In fact, the primitive group $G \cong 2$. $\mathrm{A}_{5}$ gives essentially the only example of this kind. 
The main purpose of this section is to prove the following result (cf Theorem 1.19).

Theorem 4.5 The inequality $\operatorname{lct}\left(\mathbb{P}^{3}, \bar{G}\right) \geqslant 5 / 4$ holds if the following three conditions are satisfied: the group $G$ is primitive, the group $G$ does not have semi-invariants of degree at most 4 , and the inequality $|\bar{G}| \geqslant 169$ holds.

Proof Suppose that $G$ is primitive and does not have semi-invariants of degree at most 4 , the inequality $|\bar{G}| \geqslant 169$ holds, but $\operatorname{lct}\left(\mathbb{P}^{3}, \bar{G}\right)<5 / 4$. Let us derive a contradiction.

There is an effective $\bar{G}$-invariant $\mathbb{Q}$-divisor $D$ on $\mathbb{P}^{3}$ such that $D \sim \mathbb{Q}-K_{\mathbb{P}^{3}}$ and a positive rational number $\lambda<5 / 4$ such that $\left(\mathbb{P}^{3}, \lambda D\right)$ is strictly $\log$ canonical.

Let $S$ be an irreducible subvariety in $\mathbb{P}^{3}$ that is a minimal center in $\mathbb{L} \mathbb{C}\left(\mathbb{P}^{3}, \lambda D\right)$. Then $S$ is a curve by Lemma 2.11 .

Note that $g(S) \in \mathbb{L} \mathbb{C S}\left(\mathbb{P}^{3}, \lambda D\right)$ for every $g \in \bar{G}$, because the divisor $D$ is $\bar{G}$-invariant. It follows from Lemma 2.6 that

$$
S \cap g(S) \neq \varnothing \Longleftrightarrow S=g(S)
$$

for every $g \in \bar{G}$. It follows from Lemma 2.8 that we may assume that

$$
\mathbb{L} \mathbb{C} S\left(\mathbb{P}^{3}, \lambda D\right)=\bigcup_{g \in \bar{G}}\{g(S)\} .
$$

Let $\mathcal{I}$ be the multiplier ideal sheaf of the $\log$ pair $\left(\mathbb{P}^{3}, \lambda D\right)$, and let $\mathcal{L}$ be the log canonical singularities subscheme of the log pair $\left(\mathbb{P}^{3}, \lambda D\right)$. Then there is an exact sequence

$$
0 \longrightarrow H^{0}\left(\mathcal{O}_{\mathbb{P}^{3}}(1) \otimes \mathcal{I}\right) \longrightarrow H^{0}\left(\mathcal{O}_{\mathbb{P}^{3}}(1)\right) \longrightarrow H^{0}\left(\mathcal{O}_{\mathcal{L}} \otimes \mathcal{O}_{\mathbb{P}^{3}}(1)\right) \longrightarrow 0
$$

by Theorem 2.3. Then it follows from Theorem 2.7 that $S$ is a smooth curve of genus $g$ such that $2 g-2<\operatorname{deg}(S)$.

Let $Z$ be the $\bar{G}$-orbit of the curve $S$. Then $Z$ is smooth and $\operatorname{deg}(Z) \leqslant 6$ by Lemma 2.9. Then $2 g-2<\operatorname{deg}(S) \leqslant 6$, which implies that $g \leqslant 3$. Note that $Z=\mathcal{L}$ by Remark 2.2 , because $\left(\mathbb{P}^{3}, \lambda D\right)$ is $\log$ canonical. Moreover, the curve $Z$ is not contained in a plane, because $G$ is transitive.

Let $r$ be the number of irreducible components of $Z$. Then $6 \geqslant \operatorname{deg}(Z)=r \operatorname{deg}(S)$, which implies that $r \leqslant 6$. Note that $g=0$ if $r \geqslant 3$.

Using (4.6) and the Riemann-Roch theorem, we see that

$$
4=h^{0}\left(\mathcal{O}_{\mathcal{L}} \otimes \mathcal{O}_{\mathbb{P}^{3}}(1)\right)=r(\operatorname{deg}(S)-g+1),
$$


because $\mathcal{L}=Z$ and $2 g-2<\operatorname{deg}(S)$. In particular, we see that $r \leqslant 2$.

One has $\operatorname{deg}(S) \neq 1$, because $G$ is primitive. Thus $S$ is not contained in a plane, because otherwise the $\bar{G}$-orbit of the plane spanned by $S$ would give a semi-invariant of $G$ of degree 1 or 2 . Thus, we have $6 \geqslant \operatorname{deg}(Z)=r \operatorname{deg}(S) \geqslant 3 r$.

If $r=2$, then $\operatorname{deg}(S)=3$ and $g=0$, which contradicts the equality (4.7). We see that $r=1$ and $Z=S$. Then $g \leqslant 1$ by Theorem 2.14 and Remark 2.13 , because $|\bar{G}| \geqslant 169$.

Arguing as in the proof of Theorem 4.3, we see that $g \neq 0$, because $G$ does not have semi-invariants of degree 4. Then it follows from (4.7) that $g=1$ and $\operatorname{deg}(S)=4$. We see that $S=Q_{1} \cap Q_{2}$, where $Q_{1}$ and $Q_{2}$ are irreducible quadrics in $\mathbb{P}^{3}$.

Let $\mathcal{P}$ be a pencil generated by $Q_{1}$ and $Q_{2}$. Then $\mathcal{P}$ contains exactly 4 singular surfaces, which are simple quadric cones. This means that there is a $\bar{G}$-orbit in $\mathbb{P}^{3}$ consisting of at most 4 points, which is impossible by Remark 2.10.

In the rest of this section we will refine the assertion of Theorem 4.5 by removing the assumption that $\bar{G}$ contains at least 169 elements and providing an explicit list of possible finite subgroups in $\mathrm{PGL}_{4}(\mathbb{C})$ that satisfy all hypothesis of Theorem 4.5 (cf Theorems 4.1 and 4.2). Let us start with the following example.

Example 4.8 (See Blichfeldt [3, Section 123] and Nieto [30].) Let $\mathbb{H}$ be a subgroup in $\mathrm{SL}_{4}(\mathbb{C})$ that is conjugate to the subgroup generated by

$$
\left(\begin{array}{llll}
0 & 0 & 1 & 0 \\
0 & 0 & 0 & 1 \\
1 & 0 & 0 & 0 \\
0 & 1 & 0 & 0
\end{array}\right), \quad\left(\begin{array}{llll}
0 & 1 & 0 & 0 \\
1 & 0 & 0 & 0 \\
0 & 0 & 0 & 1 \\
0 & 0 & 1 & 0
\end{array}\right), \quad\left(\begin{array}{cccc}
1 & 0 & 0 & 0 \\
0 & 1 & 0 & 0 \\
0 & 0 & -1 & 0 \\
0 & 0 & 0 & -1
\end{array}\right), \quad\left(\begin{array}{cccc}
1 & 0 & 0 & 0 \\
0 & -1 & 0 & 0 \\
0 & 0 & 1 & 0 \\
0 & 0 & 0 & -1
\end{array}\right),
$$

and let $N \subset \mathrm{SL}_{4}(\mathbb{C})$ be the normalizer of the subgroup $\mathbb{H}$. There is an exact sequence of groups ${ }^{3}$

$$
1 \longrightarrow \tilde{\mathbb{H}} \stackrel{\alpha}{\longrightarrow} N \stackrel{\beta}{\longrightarrow} \mathrm{S}_{6} \longrightarrow 1,
$$

where $\widetilde{\mathbb{H}}=\langle\mathbb{H}, \operatorname{diag}(\sqrt{-1})\rangle$. One can show that $N$ is a primitive subgroup of $\mathrm{SL}_{4}(\mathbb{C})$.

${ }^{3}$ The choice of the epimorphism $\beta$ is not canonical even up to conjugation, due to the existence of outer automorphisms of $\mathrm{S}_{6}$. There are essentially two possible choices of $\beta$. To fix one of them we use the fact that the subspace $W \subset \operatorname{Sym}^{4}\left(\mathbb{C}^{4}\right)$ of $\widetilde{\mathbb{H}}$-invariant quartics is five-dimensional; moreover, the group $N / \widetilde{\mathbb{H}}$ acts on $W$, and $W$ is an irreducible representation of $N / \widetilde{\mathbb{H}}$ (cf the proof of Lemma 4.12 and references therein). We choose $\beta$ so that $W$ corresponds to the standard five-dimensional representation of $\mathrm{S}_{6}$ twisted by the sign representation. Another way to describe the choice of $\beta$ is through introducing the action of $N / \widetilde{\mathbb{H}}$ on the space $W^{\prime}=\Lambda^{2}\left(\mathbb{C}^{4}\right)$ (see [30]). 
The following theorem provides an explicit list of possible finite subgroups in $\mathrm{PGL}_{4}(\mathbb{C})$ that satisfy all hypotheses of Theorem 4.5:

Theorem 4.9 (See [3, Chapter VII; 14, Section 8.5].) Let $G$ be a primitive subgroup of $\mathrm{SL}_{4}(\mathbb{C})$ such that $Z(G) \subseteq[G, G]$. Then one of the following possibilities holds:

- either $G$ satisfies the hypotheses of Lemma 3.24 or Lemma 3.26,

- or $G$ is one of the following groups:

- $\mathrm{A}_{5}$ or $\mathrm{S}_{5}$,

- $\mathrm{SL}_{2}\left(\mathbb{F}_{5}\right)$,

- $\mathrm{SL}_{2}\left(\mathbb{F}_{7}\right)$,

- 2. $\mathrm{A}_{6}$, which is a central extension of the group $\mathrm{A}_{6} \cong \bar{G}$,

- $2 . \mathrm{S}_{6}$, which is a central extension ${ }^{4}$ of the group $\mathrm{S}_{6} \cong \bar{G}$,

- 2. $\mathrm{A}_{7}$, which is a central extension of the group $\mathrm{A}_{7} \cong \bar{G}$,

$-\mathrm{Sp}_{4}\left(\mathbb{F}_{3}\right)$,

- in the notation of Example 4.8, a primitive subgroup in $N$ that contains $\alpha(\tilde{\mathbb{H}})$.

It should be pointed out that Theorem 4.9 describes primitive subgroups of $\mathrm{SL}_{4}(\mathbb{C})$ up to conjugation. Namely, if there are two monomorphisms $\iota_{1}: G \rightarrow \mathrm{SL}_{4}(\mathbb{C})$ and $\iota_{2}: G \rightarrow \mathrm{SL}_{4}(\mathbb{C})$ such that both subgroups $\iota_{1}(G)$ and $\iota_{2}(G)$ are primitive, then it follows from [3, Chapter VII] that $\iota_{1}(G)$ and $\iota_{2}(G)$ are conjugate, but it may happen that the representations of the group $G$ given by $\iota_{1}$ and $\iota_{2}$ are nonisomorphic, ie there is no element $g \in \mathrm{SL}_{4}(\mathbb{C})$ that makes the diagram

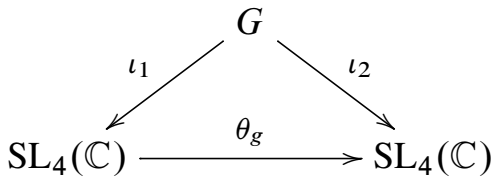

commutative, where $\theta_{g}$ is the conjugation by $g$ (cf [13]).

Lemma 4.10 Suppose that $G \cong 2 . \mathrm{A}_{6}$. Then $G$ has no semi-invariants of degree at most 4 .

Proof Semi-invariants of $G$ are its invariants by Remark 1.15, and $G$ has no odd degree invariants, because $G$ contains a scalar matrix whose nonzero entries are -1 . To complete the proof, it is enough to prove that $G$ has no invariants of degree 4 .

\footnotetext{
${ }^{4}$ There are three nonisomorphic nontrivial central extensions of the group $\mathrm{S}_{6}$ with the center isomorphic to $\mathbb{Z}_{2}$, two of which are embedded in $\mathrm{SL}_{4}(\mathbb{C})$ (cf [13]). But up to conjugation there is only one subgroup of $\mathrm{PGL}_{4}(\mathbb{C})$ isomorphic to $\mathrm{S}_{6}$.
} 
Let $V \cong \mathbb{C}^{4}$ be the irreducible representation of the group $G$ that corresponds to the embedding $G \subset \mathrm{SL}_{4}(\mathbb{C})$. Without loss of generality, we may assume that $\Lambda^{2} V \cong \mathbb{C}^{6}$ is a permutation representation of the group $G / Z(G) \cong \mathrm{A}_{6}$, because $G$ has two fourdimensional irreducible representations, which give one subgroup $G \subset \mathrm{SL}_{4}(\mathbb{C})$ up to conjugation.

Let $\chi$ be the character of the representation $V$, and let $\chi_{4}$ be the character of the representation $\operatorname{Sym}^{4}(V)$. Then

$$
\chi_{4}(g)=\frac{1}{24}\left(\chi(g)^{4}+6 \chi(g)^{2} \chi\left(g^{2}\right)+3 \chi\left(g^{2}\right)^{2}+8 \chi(g) \chi\left(g^{3}\right)+6 \chi\left(g^{4}\right)\right)
$$

for every $g \in G$. The values of the characters $\chi$ and $\chi_{4}$ are listed in Table 1 . In this

\begin{tabular}{|c|c|c|c|c|c|c|c|c|c|c|}
\hline & {$[5,1]_{10}$} & {$[5,1]_{5}$} & {$[4,2]_{8}$} & {$[3,3]_{6}$} & {$[3,3]_{3}$} & {$[3,1,1,1]_{6}$} & {$[3,1,1,1]_{3}$} & {$[2,2,1,1]_{4}$} & $z$ & $e$ \\
\hline$\#$ & 144 & 144 & 180 & 40 & 40 & 40 & 40 & 90 & 1 & 1 \\
\hline$\chi$ & 1 & -1 & 0 & -1 & 1 & 2 & -2 & 0 & -4 & 4 \\
\hline$\chi_{4}$ & 0 & 0 & -1 & 2 & 2 & -4 & -4 & 3 & 35 & 35 \\
\hline
\end{tabular}

Table 1

table, the first row lists the types of the elements in $G$ (for example, the symbol $[5,1]_{10}$ denotes the set ${ }^{5}$ of order 10 elements whose image in $\mathrm{A}_{6}$ is a product of disjoint cycles of length 5 and 1), and $z$ and $e$ are the nontrivial element in the center of $G$ and the identity element, respectively.

Now one can check that the inner product of the character $\chi_{4}$ and the trivial character is zero, which implies that the subgroup $G$ does not have invariants of degree 4 .

Lemma 4.11 If $G \cong 2 . \mathrm{S}_{6}$ or $G \cong 2 . \mathrm{A}_{7}$, then $G$ has no semi-invariants of degree at most 4 .

Proof Recall that these groups contain 2. $\mathrm{A}_{6}$ and we can apply Lemma 4.10.

Lemma 4.12 Under the assumptions of Theorem 4.9 the subgroup $G$ has no semiinvariants of degree at most 4 if and only if $G$ is one of the following groups:

- $2 . \mathrm{A}_{6}, 2 . \mathrm{S}_{6}$ or $2 . \mathrm{A}_{7}$,

- $\mathrm{Sp}_{4}\left(\mathbb{F}_{3}\right)$,

${ }^{5}$ Note that these sets do not coincide with conjugacy classes. For example, the image of the set of the elements of type $[5,1]_{10}$ under the natural projection 2. $\mathrm{A}_{6} \rightarrow \mathrm{A}_{6}$ is a union of two different conjugacy classes in $\mathrm{A}_{6}$. 
- in the notation of Example 4.8, a subgroup of $N$ that satisfies one of the following four conditions:

- $G=N$,

- $\alpha(\tilde{\mathbb{H}}) \subsetneq G$ and $\beta(G) \cong \mathrm{A}_{6}$,

- $\alpha(\widetilde{\mathbb{H}}) \subsetneq G$ and $\beta(G) \cong \mathrm{S}_{5}$, where the embedding $\beta(G) \subset \mathrm{S}_{6}$ is nonstandard, ie the standard one twisted by an outer automorphism of $\mathrm{S}_{6}$,

- $\alpha(\widetilde{\mathbb{H}}) \subsetneq G$ and $\beta(G) \cong \mathrm{A}_{5}$, where the embedding $\beta(G) \subset \mathrm{S}_{6}$ is nonstandard.

Proof Let $d$ be the smallest positive number such $G$ has an semi-invariant of degree $d$. If $G \cong 2 . \mathrm{A}_{6}$, then $d \geqslant 5$ by Lemma 4.10 . If $G \cong 2 . \mathrm{S}_{6}$ or $G \cong 2 . \mathrm{A}_{7}$, then $d \geqslant 5$ by Lemma 4.11. In fact, one can check by direct computation that $d=8$ if $G \cong 2$ A $_{6}$ or $G \cong 2 . \mathrm{S}_{6}$ or $G \cong 2 \mathrm{~A}_{7}$. If $G \cong \mathrm{SL}_{2}\left(\mathbb{F}_{7}\right)$, then the equality $d=4$ holds by [26] and Remark 1.15. If $G \cong \operatorname{Sp}_{4}\left(\mathbb{F}_{3}\right)$, then the equality $d=12$ holds by [28] and Remark 1.15.

Suppose that $G \cong \mathrm{SL}_{2}\left(\mathbb{F}_{5}\right) \cong 2 \mathrm{~A}_{5}$. Then there is a $\bar{G}$-invariant smooth rational cubic curve $C \subset \mathbb{P}^{3}$, because the representation $G \rightarrow \mathrm{GL}_{4}(\mathbb{C})$ is a symmetric square of a two-dimensional representation of the group $G$. The surface swept out by the lines tangent to the curve $C$ is a $\bar{G}$-invariant surface of degree 4 (cf proof of Theorem 4.3). Therefore, the inequality $d \leqslant 4$ holds $^{6}$.

Let us use the notation of Example 4.8. By Theorem 4.9, Remark 2.12 and Lemmas 3.24 and 3.26, to complete the proof we may assume that $G$ is a primitive subgroup in $N$ that contains $\alpha(\widetilde{\mathbb{H}})$.

One can show that the group $\widetilde{\mathbb{H}}$ has no invariants of degree less than 4 and its invariants of degree 4 form a five-dimensional vector space $W$ (see eg [33, Lemma 3.18]).

The group $\beta(G)$ naturally acts on $W$. Moreover, the subgroup $G$ has an invariant of degree 4 if and only if the representation $W$ has a one-dimensional subrepresentation of the group $\beta(G)$. On the other hand, it follows from [30] that if $G=N$, then $W$ is an irreducible representation of $\beta(G)=\mathrm{S}_{6}$.

It follows from [3, Section 123] that, up to conjugation, there exist exactly 9 possibilities for the subgroup $G \subset N$ such that $G$ is primitive. These possibilities are listed in Table 2. In this table, the first column lists the labels of the subgroup $G$ according to [3, Section 123] and the last column lists the dimensions of the irreducible $\beta(G)-$ subrepresentations of $W$.

Note that $\mathbb{H} \subset \widetilde{\mathbb{H}}$ has no semi-invariants of degree 3 , because $\mathbb{H}$ has no invariants of degree 3 , the center of the group $\mathbb{H}$ coincides with its commutator and acts nontrivially on cubic forms.

\footnotetext{
${ }^{6}$ Actually, one can show that $d=4$ in this case.
} 


\begin{tabular}{|c|c|c|c|}
\hline Label of the group $G$ & $\beta(G)$ & $\begin{array}{c}\text { Generators of the } \\
\text { subgroup } \beta(G) \subseteq \mathrm{S}_{6}\end{array}$ & Splitting type \\
\hline $13^{\circ}$ & $\mathbb{Z}_{5}$ & $(24635)$ & $1,1,1,1,1$ \\
\hline $14^{\circ}$ & $\mathbb{Z}_{5} \rtimes \mathbb{Z}_{2}$ & $(24635),(36)(45)$ & $1,2,2$ \\
\hline $15^{\circ}$ & $\mathbb{Z}_{5} \rtimes \mathbb{Z}_{4}$ & $(24635),(3465)$ & $1,2,2$ \\
\hline $16^{\circ}$ & $\mathrm{A}_{5}$ & $(24635),(34)(56)$ & 1,4 \\
\hline $17^{\circ}$ & $\mathrm{A}_{5}$ & $(24635),(12)(36)$ & 5 \\
\hline $18^{\circ}$ & $\mathrm{S}_{5}$ & $(24635),(56)$ & 1,4 \\
\hline $19^{\circ}$ & $\mathrm{S}_{5}$ & $(24635),(12)(34)(56)$ & 5 \\
\hline $20^{\circ}$ & $\mathrm{A}_{6}$ & $(24635),(12)(34)$ & 5 \\
\hline $21^{\circ}$ & $\mathrm{S}_{6}$ & $(24635),(12)$ & 5 \\
\hline
\end{tabular}

Table 2

The subgroups of $N$ described in Lemma 4.12 are the subgroups $21^{\circ}, 20^{\circ}, 19^{\circ}, 17^{\circ}$, respectively. We see that $d \leqslant 4$ if $G$ is the subgroup $13^{\circ}, 14^{\circ}, 15^{\circ}, 16^{\circ}$ or $18^{\circ}$. On the other hand, if $G$ is the subgroup $17^{\circ}, 19^{\circ}, 20^{\circ}$ or $21^{\circ}$, then the subgroup $G$ has neither semi-invariants of degree less than 4 , nor invariants of degree 4 . Let us prove that the subgroup $17^{\circ}$ does not have semi-invariants of degree 4 . Since the absence of semi-invariants of degree 4 implies the absence of semi-invariants of degree 2 , this would imply that in the case when $G$ is the subgroup $17^{\circ}, 19^{\circ}, 20^{\circ}$ or $21^{\circ}$ of the group $N$ the inequality $d \geqslant 5$ holds $^{7}$.

Suppose that $G$ is the subgroup $17^{\circ}$, and suppose, in addition, that $G$ does have a semiinvariant $\Phi$ of degree 4 . Let us show that this assumption leads to a contradiction.

Note that the polynomial $\Phi$ is not $\widetilde{\mathbb{H}}$-invariant, because $\Phi$ is not $G$-invariant and $G / \widetilde{\mathbb{H}} \cong \beta(G) \cong \mathrm{A}_{5}$ is a simple group. Let $Z$ be the center of the group $\widetilde{\mathbb{H}}$. Put $\overline{\mathbb{H}}=\phi(\widetilde{\mathbb{H}})$. Then $\widetilde{\mathbb{H}} / Z \cong \overline{\mathbb{H}} \cong \mathbb{Z}_{2}^{4}$, and $Z$ acts trivially on $\Phi$. Thus, there is a homomorphism $\xi: \overline{\mathbb{H}} \rightarrow \mathbb{C}^{*}$ such that $\operatorname{ker}(\xi) \neq \overline{\mathbb{H}}$, which implies that $\operatorname{ker}(\xi) \cong \mathbb{Z}_{2}^{3}$, because $\operatorname{im}(\chi)$ is a cyclic group. Let $\theta: \bar{G} \rightarrow \operatorname{Aut}(\overline{\mathbb{H}})$ be the homomorphism such that

$$
\theta(g)(h)=g h g^{-1} \in \overline{\mathbb{H}} \cong \mathbb{Z}_{2}^{4}
$$

for all $g \in \bar{G}$ and $h \in \overline{\mathbb{H}}$. Consider $\overline{\mathbb{H}}$ as a vector space over $\mathbb{F}_{2}$. Then $\theta$ induces a monomorphism $\tau: \beta(G) \rightarrow \mathrm{GL}_{4}\left(\mathbb{F}_{2}\right)$ and $\operatorname{ker}(\xi)$ is a $\operatorname{im}(\tau)$-invariant subspace. But $\operatorname{im}(\tau) \cong \mathrm{A}_{5}$ has no nontrivial three-dimensional representations over $\mathbb{F}_{2}$, because

\footnotetext{
${ }^{7}$ In fact, one can check by direct computation that $d=8$ if $G$ is the subgroup $17^{\circ}, 19^{\circ}, 20^{\circ}$ or $21^{\circ}$.
} 
$\left|\mathrm{GL}_{3}\left(\mathbb{F}_{2}\right)\right|=168$ is not divisible by $\left|\mathrm{A}_{5}\right|=60$. Thus, we see that there is a nonzero element $t \in \overline{\mathbb{H}}$ such that $t$ is $\operatorname{im}(\tau)$-invariant. Let $F$ be the stabilizer of $t$ in $\mathrm{GL}_{4}\left(\mathbb{F}_{2}\right)$. Then $\mathrm{A}_{5} \cong \operatorname{im}(\tau) \subset F$, which is impossible, because $|F|=1344$ is not divisible by $\left|\mathrm{A}_{5}\right|=60$.

Combining the previous results we obtain the following.

Theorem 4.13 Let $G$ be a finite subgroup in $\mathrm{SL}_{4}(\mathbb{C})$. Then the following conditions are equivalent:

- The singularity $(V \ni O)$ is exceptional.

- The inequality $\operatorname{lct}\left(\mathbb{P}^{3}, \bar{G}\right) \geqslant 5 / 4$ holds.

- The group $G$ is primitive and $G$ does not have semi-invariants of degree at most 4 .

- $\bar{G}=\phi\left(G^{\prime}\right)$, where $G^{\prime}$ is one of the 8 subgroups listed in Lemma 4.12.

Proof This follows from Theorems 1.17, 4.5 and 4.9 and Lemma 4.12.

\section{Five-dimensional case}

The purpose of this section is to present an explicit classification of exceptional fivedimensional singularities (see Theorem 5.6, cf Theorems 4.1, 4.2 and 4.13), and prove some relevant results.

Let $\bar{G}$ be a finite subgroup in $\operatorname{Aut}\left(\mathbb{P}^{4}\right)$, and consider the natural projection

$$
\phi: \mathrm{SL}_{5}(\mathbb{C}) \rightarrow \operatorname{Aut}\left(\mathbb{P}^{4}\right) \cong \operatorname{PGL}_{5}(\mathbb{C}) .
$$

Then there is a finite subgroup $G \subset \mathrm{SL}_{5}(\mathbb{C})$ such that $\phi(G)=\bar{G}$. Suppose that $G$ is primitive. Then we may assume that $Z(G) \subseteq[G, G]$ (see $[5 ; 14]$ ).

Example 5.1 (cf Appendix A) Let $\mathbb{H}$ be the Heisenberg group of all unipotent $(3 \times 3)$-matrices with entries in $\mathbb{F}_{5}$. Then there is a monomorphism $\rho: \mathbb{H} \rightarrow \operatorname{SL}_{5}(\mathbb{C})$. Let $\mathbb{H M}$ be the normalizer of the subgroup $\rho(\mathbb{H}) \subset \mathrm{SL}_{5}(\mathbb{C})$. Then there is an exact sequence

$$
1 \longrightarrow \mathbb{H} \stackrel{\alpha}{\longrightarrow} \mathbb{H} \mathbb{M} \stackrel{\beta}{\longrightarrow} \operatorname{SL}_{2}\left(\mathbb{F}_{5}\right) \longrightarrow 1,
$$

and $\mathbb{H} \mathbb{M}$ is a primitive subgroup in $\mathrm{SL}_{5}(\mathbb{C})$ (see $[5$, Theorem 9A; 17]). 
Theorem 5.2 (See [5; 14, Section 8.5].) Let $G$ be a finite primitive subgroup in $\mathrm{SL}_{5}(\mathbb{C})$ such that $Z(G) \subseteq[G, G]$. Then $G$ is one of the groups $\mathrm{A}_{5}, \mathrm{~A}_{6}, \mathrm{~S}_{5}, \mathrm{~S}_{6}$, $\mathrm{PSL}_{2}\left(\mathbb{F}_{11}\right), \mathrm{PSp}_{4}\left(\mathbb{F}_{3}\right)$, or, in the notation of Example 5.1, a primitive subgroup of $\mathbb{H} \mathbb{M}$ that contains $\alpha(\mathbb{H})$.

Note that if there are two monomorphisms $\iota_{1}: G \rightarrow \mathrm{SL}_{5}(\mathbb{C})$ and $\iota_{2}: G \rightarrow \mathrm{SL}_{5}(\mathbb{C})$ such that both subgroups $\iota_{1}(G)$ and $\iota_{2}(G)$ are primitive, then $\iota_{1}(G)$ and $\iota_{2}(G)$ are conjugate.

Lemma 5.3 Suppose that $G$ is one of the following groups: $\mathrm{A}_{5}, \mathrm{~A}_{6}, \mathrm{~S}_{5}, \mathrm{~S}_{6}$, $\mathrm{PSL}_{2}\left(\mathbb{F}_{11}\right)$ or $\mathrm{PSp}_{4}\left(\mathbb{F}_{3}\right)$. Then $G$ has an invariant of degree at most 4 , which implies that $\operatorname{lct}\left(\mathbb{P}^{4}, \bar{G}\right) \leqslant 4 / 5$.

Proof If $G$ is $\mathrm{A}_{5}, \mathrm{~A}_{6}, \mathrm{~S}_{5}$ or $\mathrm{S}_{6}$, then $G$ has an invariant of degree 2 by Remark 2.12. If $G \cong \operatorname{PSp}_{4}\left(\mathbb{F}_{3}\right)$, then $G$ has an invariant of degree 4 (see [7]). If $G \cong \operatorname{PSL}_{2}\left(\mathbb{F}_{11}\right)$, then $G$ has an invariant of degree 3 (see [1]).

Lemma 5.4 In the notation of Example 5.1, suppose that $\alpha(\mathbb{H}) \subsetneq G \subseteq \mathbb{H} \mathbb{M}$. Then $G$ has no semi-invariants of degree at most 5 if and only if either $G=\mathbb{H M}$ or $G$ is a subgroup of $\mathbb{H} \mathbb{M}$ of index 5 .

Proof Let $V$ be the vector space of $\mathbb{H}$-invariant forms of degree 5. Then the group $\mathbb{H M} / \alpha(\mathbb{H}) \cong \mathrm{SL}_{2}\left(\mathbb{F}_{5}\right) \cong 2 . \mathrm{A}_{5}$ naturally acts on the vector space $V$. Moreover, it follows from [17, Theorem 3.5] that $V=V^{\prime} \oplus V^{\prime \prime}$, where $V^{\prime}$ and $V^{\prime \prime}$ are threedimensional $\operatorname{im}(\beta)$-invariant linear subspaces that arise from two nonequivalent threedimensional representations of the group $\mathrm{A}_{5}$, respectively. Therefore, we see that $G$ has a semi-invariant of degree 5 if and only if $V^{\prime}$ has a $\beta(G)$-invariant one-dimensional subspace.

Let $Z \cong \mathbb{Z}_{2}$ be the center of the group $\mathbb{H M} / \alpha(\mathbb{H}) \cong 2 . \mathrm{A}_{5}$. Then $2 . \mathrm{A}_{5} / Z \cong \mathrm{A}_{5}$. Moreover, either $\beta(G)$ is cyclic, or $Z \subseteq \beta(G)$ and $\beta(G) / Z$ is one of the following subgroups of $\mathrm{A}_{5}$ : dihedral group of order 6 , dihedral group of order 10 , the group $\mathbb{Z}_{2} \times \mathbb{Z}_{2}$, the group $A_{4}$, the group $A_{5}$.

If $\beta(G)$ is cyclic, then $V^{\prime}$ is a sum of one-dimensional $\beta(G)$-invariant linear subspaces. Hence we may assume that $Z \subseteq \beta(G)$. Recall that $Z \cong \mathbb{Z}_{2}$ acts trivially on $V^{\prime}$. Thus, if $\beta(G) / Z \cong \mathbb{Z}_{2} \times \mathbb{Z}_{2}$, then $V^{\prime}$ is a sum of one-dimensional $\beta(G)$-invariant subspaces.

If $\beta(G) / Z$ is a dihedral group, then $V^{\prime}$ must have one-dimensional $\beta(G)$-invariant subspace, because irreducible representations of dihedral groups are one-dimensional or two-dimensional. 
If $\beta(G) / Z \cong \mathrm{A}_{5}$ or $\beta(G) / Z \cong \mathrm{A}_{4}$, then $V^{\prime}$ is an irreducible representation of $\beta(G) / Z$, which implies that $V^{\prime}$ is an irreducible representation of the group $\beta(G)$. Now using Corollary A.2, we complete the proof.

The main purpose of this section is to prove the following result.

Theorem 5.5 In the notation of Example 5.1, let $G$ be a subgroup of the group $\mathbb{H} \mathbb{M}$ of index 5 . Then $\operatorname{lct}\left(\mathbb{P}^{4}, \bar{G}\right) \geqslant 6 / 5$.

Combining the previous results we obtain the following.

Theorem 5.6 Let $G$ be a finite subgroup in $\mathrm{SL}_{5}(\mathbb{C})$. Then the following conditions are equivalent:

- The singularity $(V \ni O)$ is exceptional.

- The inequality $\operatorname{lct}\left(\mathbb{P}^{4}, \bar{G}\right) \geqslant 6 / 5$ holds.

- The group $G$ is primitive and $G$ does not have semi-invariants of degree at most 5 .

- In the notation of Example 5.1, either $G \cong \mathbb{H} \mathbb{M}$ or $G$ is isomorphic to a subgroup of the group $\mathbb{H} \mathbb{M}$ of index 5 .

Proof The required assertion follows from Theorems 1.17, 5.5, 5.2 and Lemmas 5.4 and 5.3.

In the remaining part of this section we will prove Theorem 5.5. Let us use the notation of Example 5.1. Suppose that $G$ be a subgroup of the group $\mathbb{H} \mathbb{M}$ of index 5 .

Lemma 5.7 Let $\Lambda$ be a $\bar{G}$-invariant subset of $\mathbb{P}^{4}$. Then $\Lambda$ consists of at least 10 points.

Proof The required assertion follows from Lemma 5.4 and Corollary A.2.

Suppose that $\operatorname{lct}\left(\mathbb{P}^{4}, \bar{G}\right)<6 / 5$. Let us derive a contradiction.

There is a rational positive number $\lambda<6 / 5$ and an effective $\bar{G}$-invariant $\mathbb{Q}$-divisor $D$ on $\mathbb{P}^{5}$ such that $D \sim \mathbb{Q}-K_{\mathbb{P}^{4}}$ and the $\log$ pair $\left(\mathbb{P}^{4}, \lambda D\right)$ is strictly log canonical. Let $S$ be an irreducible subvariety of $\mathbb{P}^{4}$ that is a minimal center in $\mathbb{L} \mathbb{C}\left(\mathbb{P}^{4}, \lambda D\right)$. Then $S$ is either a curve or a surface by Lemma 2.11 . 
Let $Z$ be the $\bar{G}$-orbit of the subvariety $S \subset \mathbb{P}^{4}$, and let $r$ be the number of irreducible components of the subvariety $Z$. We may assume that

$$
\mathbb{L} \mathbb{C S}\left(\mathbb{P}^{4}, \lambda D\right)=\bigcup_{g \in \bar{G}}\{g(S)\}
$$

by Lemma 2.8. Then $\operatorname{Supp}(Z)=\operatorname{LCS}\left(\mathbb{P}^{4}, \lambda D\right)$. It follows from Lemma 2.6 that

$$
S \cap g(S) \neq \varnothing \Longleftrightarrow S=g(S)
$$

for every $g \in \bar{G}$. Then $\operatorname{deg}(Z)=r \operatorname{deg}(S)$.

Let $\mathcal{I}$ be the multiplier ideal sheaf of the $\log$ pair $\left(\mathbb{P}^{4}, \lambda D\right)$, and let $\mathcal{L}$ be the log canonical singularities subscheme of the $\log$ pair $\left(\mathbb{P}^{4}, \lambda D\right)$. By Theorem 2.3 , there is an exact sequence

$$
0 \longrightarrow H^{0}\left(\mathcal{O}_{\mathbb{P}^{4}}(n) \otimes \mathcal{I}\right) \longrightarrow H^{0}\left(\mathcal{O}_{\mathbb{P}^{4}}(n)\right) \longrightarrow H^{0}\left(\mathcal{O}_{\mathcal{L}} \otimes \mathcal{O}_{\mathbb{P}^{4}}(n)\right) \longrightarrow 0
$$

for every $n \geqslant 1$. Note that $Z=\mathcal{L}$ by Remark 2.2.

Lemma 5.9 The center $S$ is not a curve.

Proof Suppose that $S$ is a curve. Then it follows from Theorem 2.7 that $S$ is a smooth curve of genus $g$ such that $2 g-2<\operatorname{deg}(S)$. Moreover, it follows from Lemma 2.9 that $\operatorname{deg}(Z) \leqslant 10$. Then $2 g-2<\operatorname{deg}(S) \leqslant 10$, which implies that $g \leqslant 5$. The curve $Z$ is not contained in a hyperplane, because $G$ is transitive. Then $10 \geqslant \operatorname{deg}(Z)=r \operatorname{deg}(S)$, which implies that $r \leqslant 10$.

Using (5.8) and the Riemann-Roch theorem, we see that

$$
5=h^{0}\left(\mathcal{O}_{\mathcal{L}} \otimes \mathcal{O}_{\mathbb{P}^{3}}(1)\right)=r(\operatorname{deg}(S)-g+1),
$$

because $\mathcal{L}=Z$ and $2 g-2<\operatorname{deg}(S)$. Thus, either $r=1$ or $r=5$.

If $r=5$, then $\operatorname{deg}(S)=2$ and $g=0$, which contradicts (5.10). We see that $r=1$. Thus $S$ is a $\bar{G}$-invariant irreducible curve of genus $g \leqslant 5$, which is impossible by Lemma A.8.

We see that $S$ is a surface. Then $\operatorname{deg}(Z) \leqslant 10$ by Lemma 2.9. It follows from Theorem 2.7 that $S$ is normal and has at most rational singularities, and there is an effective $\mathbb{Q}$-divisor $B_{S}$ and an ample $\mathbb{Q}$-divisor $\Delta$ on the surface $S$ such that

$$
K_{S}+B_{S}+\left.\Delta \sim \mathbb{Q} \mathcal{O}_{\mathbb{P}^{4}}(1)\right|_{S},
$$

and the log pair $\left(S, B_{S}\right)$ has Kawamata log terminal singularities. Therefore, the equality $r=1$ holds, since two irreducible surfaces in $\mathbb{P}^{4}$ have nonempty intersection. Thus, we see that the surface $S=Z$ is $\bar{G}$-invariant. 
Lemma 5.11 The surface $S$ is not contained in a hyperplane in $\mathbb{P}^{4}$.

Proof The required assertion follows from the fact that $G$ is transitive.

Lemma 5.12 The surface $S$ is not contained in a quadric hypersurface in $\mathbb{P}^{4}$.

Proof Suppose that there is a quadric hypersurface $Q \subset \mathbb{P}^{4}$ such that $S \subset Q$. Then $Q$ is irreducible by Lemma 5.11. Moreover, it follows from Lemma 5.4 that there is a quadric hypersurface $Q^{\prime} \subset \mathbb{P}^{4}$ such that $S \subseteq Q \cap Q^{\prime}$, because otherwise the quadric $Q$ would be $\bar{G}$-invariant. Then $Q^{\prime}$ is irreducible by Lemma 5.11 .

Suppose that $S=Q \cap Q^{\prime}$. If $S$ is nonsingular, consider a pencil $\mathcal{P}$ generated by the quadrics $Q$ and $Q^{\prime}$. Then $\mathcal{P}$ contains exactly 5 singular quadrics, which are simple quadric cones. This means that there is a $\bar{G}$-orbit in $\mathbb{P}^{4}$ consisting of at most 5 points, which is impossible, because $G$ has no semi-invariants of degree up to 5. Therefore, the surface $S$ is singular.

It follows from [16] that $|\operatorname{Sing}(S)| \leqslant 4$, because $S$ has canonical singularities since $S$ is a complete intersection that has Kawamata log terminal singularities. But $\operatorname{Sing}(S)$ is $\bar{G}$-invariant, which contradicts Lemma 5.7.

We see that $S \neq Q \cap Q^{\prime}$. Therefore, it follows from Lemma 5.11 that either $S$ is a cone over a smooth rational cubic curve, or $S$ is a smooth cubic scroll.

If $S$ is a cone, then its vertex is $\bar{G}$-invariant, which is impossible since $G$ is transitive. Thus, we see that $S$ is a smooth cubic scroll. Then there is a unique line $L \subset S$ such that $L^{2}=-1$, which implies that $L$ must be $\bar{G}$-invariant, which is again impossible, because $G$ is transitive.

Let $H$ be a hyperplane section of the surface $S \subset \mathbb{P}^{4}$.

Lemma 5.13 The equalities $H \cdot H=-H \cdot K_{S}=5$ and $\chi\left(\mathcal{O}_{S}\right)=0$ hold.

Proof It follows from Corollary A.2 that there is $m \geqslant 0$ such that $h^{0}\left(\mathcal{O}_{\mathbb{P}^{4}}(3) \otimes \mathcal{I}\right)=5 m$. Let us show that this is possible only if $H \cdot H=-H \cdot K_{S}=5$ and $\chi\left(\mathcal{O}_{S}\right)=0$.

It follows from the Riemann-Roch theorem and Theorem 2.3 that

$$
h^{0}\left(\mathcal{O}_{S}(n H)\right)=\chi\left(\mathcal{O}_{S}(n H)\right)=\chi\left(\mathcal{O}_{S}\right)+\frac{n^{2}}{2}(H \cdot H)-\frac{n}{2}\left(H \cdot K_{S}\right)
$$

for any $n \geqslant 1$. It follows from Lemma 5.11, the equality (5.14) and the exact sequence (5.8) that

$$
5=h^{0}\left(\mathcal{O}_{S}(H)\right)=\chi\left(\mathcal{O}_{S}\right)+\frac{1}{2}(H \cdot H)-\frac{1}{2}\left(H \cdot K_{S}\right),
$$


and it follows from Lemma 5.12, the equality (5.14) and the exact sequence (5.8) that

$$
15=h^{0}\left(\mathcal{O}_{S}(2 H)\right)=\chi\left(\mathcal{O}_{S}\right)+2(H \cdot H)-\left(H \cdot K_{S}\right) .
$$

It follows from Lemmas $2.9,5.11$ and 5.12 that $4 \leqslant H \cdot H=\operatorname{deg}(S) \leqslant 10$.

Suppose that $H \cdot H=10$. It follows from the equalities (5.15) and (5.16) that $\chi\left(\mathcal{O}_{S}\right)=5$ and $H \cdot K_{S}=H \cdot H=10$, which is impossible, because $H \sim_{\mathbb{Q}} K_{S}+B_{S}+\Delta$, where $\Delta$ is ample and $B_{S}$ is effective. Thus $H \cdot H \leqslant 9$.

It follows from the equalities (5.15) and (5.16) that

$$
H \cdot K_{S}=3 \chi\left(\mathcal{O}_{S}\right)-5=3(H \cdot H)-20 .
$$

It follows from the equality (5.14) and the exact sequence (5.8) that $h^{0}\left(\mathcal{O}_{\mathbb{P}^{4}}(3) \otimes \mathcal{I}\right)=35-h^{0}\left(\mathcal{O}_{S}(3 H)\right)=35-\left(\chi\left(\mathcal{O}_{S}\right)+\frac{9}{2}(H \cdot H)-\frac{3}{2}\left(H \cdot K_{S}\right)\right)=5 m$, which implies that $H \cdot H=5, \chi\left(\mathcal{O}_{S}\right)=0$ and $H \cdot K_{S}=-5$, because $4 \leqslant H \cdot H \leqslant 9$.

Let $\pi: U \rightarrow S$ be the minimal resolution of the surface $S$. Then $\kappa(U)=-\infty$ and

$$
1-h^{1}\left(\mathcal{O}_{U}\right)=1-h^{1}\left(\mathcal{O}_{S}\right)=h^{2}\left(\mathcal{O}_{S}\right)=h^{2}\left(\mathcal{O}_{U}\right)=h^{0}\left(\mathcal{O}_{U}\left(K_{U}\right)\right)=0,
$$

because $S$ has rational singularities and $\kappa(U)=-\infty$ since $H \cdot K_{S}=-5<0$.

Corollary 5.17 The surface $S$ is birational to $E \times \mathbb{P}^{1}$, where $E$ is smooth elliptic curve.

By Remark 2.13, there is a monomorphism $\xi: \bar{G} \rightarrow \operatorname{Aut}(Y)$, which contradicts Corollary A.11.

The obtained contradiction completes the proof of Theorem 5.5.

\section{Appendix A Horrocks-Mumford group}

Let $\mathbb{H}$ be the Heisenberg group of all unipotent $(3 \times 3)$-matrices with entries in $\mathbb{F}_{5}$. Then

$$
\mathbb{H}=\left\langle x, y, z \mid x^{5}=y^{5}=z^{5}=1, x z=z x, y z=z y, x y=z y x\right\rangle
$$


for some $x, y, z \in \mathbb{H}$. There is a monomorphism $\rho: \mathbb{H} \rightarrow \mathrm{SL}_{5}(\mathbb{C})$ such that

$$
\rho(x)=\left(\begin{array}{lllll}
0 & 0 & 0 & 0 & 1 \\
1 & 0 & 0 & 0 & 0 \\
0 & 1 & 0 & 0 & 0 \\
0 & 0 & 1 & 0 & 0 \\
0 & 0 & 0 & 1 & 0
\end{array}\right), \quad \rho(y)=\left(\begin{array}{ccccc}
\zeta & 0 & 0 & 0 & 0 \\
0 & \zeta^{2} & 0 & 0 & 0 \\
0 & 0 & \zeta^{3} & 0 & 0 \\
0 & 0 & 0 & \zeta^{4} & 0 \\
0 & 0 & 0 & 0 & 1
\end{array}\right),
$$

where $\zeta$ is a nontrivial fifth root of unity. Let us identify $\mathbb{H}$ with $\operatorname{im}(\rho)$. Then $Z(\mathbb{H}) \cong \mathbb{Z}_{5}$ and

$$
\left(\begin{array}{lllll}
\zeta & 0 & 0 & 0 & 0 \\
0 & \zeta & 0 & 0 & 0 \\
0 & 0 & \zeta & 0 & 0 \\
0 & 0 & 0 & \zeta & 0 \\
0 & 0 & 0 & 0 & \zeta
\end{array}\right) \in Z(\mathbb{H})
$$

where $Z(\mathbb{H})$ is the center of $\mathbb{H}$. Let $\phi: \mathrm{GL}_{5}(\mathbb{C}) \rightarrow \mathrm{PGL}_{5}(\mathbb{C})$ be the natural projection.

Lemma A.1 [17, Section 1] Let $\chi: \mathbb{H} \rightarrow \mathrm{GL}_{N}(\mathbb{C})$ be an irreducible representation of $\mathbb{H}$. Then either $N=1$ and $Z(\mathbb{H}) \subseteq \operatorname{ker}(\chi)$, or $N$ is divisible by 5 .

Take $n \in \mathbb{Z}_{\geqslant 0}$. Then $\mathbb{H}$ naturally acts on $H^{0}\left(\mathcal{O}_{\mathbb{P}^{4}}(n)\right)$.

Corollary A.2 Let $V$ be a $\mathbb{H}$-invariant subspace in $H^{0}\left(\mathcal{O}_{\mathbb{P}^{4}}(n)\right)$. Then either $\operatorname{dim}(V)$ is divisible by 5 , or $n$ is divisible by 5 .

Let $\mathbb{H M} \subset \mathrm{SL}_{5}(\mathbb{C})$ be the normalizer of the subgroup $\mathbb{H}$. Then there is an exact sequence

$$
1 \longrightarrow \mathbb{H} \stackrel{\alpha}{\longrightarrow} \mathbb{H} \mathbb{M} \stackrel{\beta}{\longrightarrow} \operatorname{SL}_{2}\left(\mathbb{F}_{5}\right) \longrightarrow 1,
$$

and it follows from [17, Section 1] that there is a subgroup $\mathbb{M} \subset \mathbb{H} \mathbb{M}$ such that $\mathbb{H} \mathbb{M}=$ $\mathbb{H} \rtimes \mathbb{M}$ and $\mathbb{M} \cong \beta(\mathbb{M})=\operatorname{SL}_{2}\left(\mathbb{F}_{5}\right) \cong 2 . \mathrm{A}_{5}$. Put $\overline{\mathbb{H}}=\phi(\mathbb{H})$ and $\overline{\mathbb{H} M}=\phi(\mathbb{H M})$. Then $\overline{\mathbb{H M}} / \overline{\mathbb{H}} \cong \mathrm{SL}_{2}\left(\mathbb{F}_{5}\right)$ and $\overline{\mathbb{H}} \cong \mathbb{Z}_{5} \times \mathbb{Z}_{5}$. Let $Z(\mathbb{H M})$ be the center of the group $\mathbb{H} \mathbb{M}$. Then $Z(\mathbb{H M})=Z(\mathbb{H}) \cong \mathbb{Z}_{5}$.

Corollary A.3 The group $\overline{\mathbb{H M}}$ is isomorphic to $\mathbb{H} \mathbb{M} / Z(\mathbb{H M})$.

Let $G$ be a subgroup of the group $\mathbb{H} \mathbb{M}$ of index 5 . Then $G \cong \mathbb{H} \rtimes 2 . \mathrm{A}_{4} \subset \mathbb{H} \rtimes 2 . \mathrm{A}_{5}$ and $|\bar{G}|=600$, where $\bar{G}=\phi(G)$. Let $Z(G)$ be the center of the group $G$. Then $Z(G)=Z(\mathbb{H M})=Z(\mathbb{H}) \cong \mathbb{Z}_{5}$. 
Lemma A.4 Let $g$ be an element of the group $\bar{G}$ such that $g h=h g \in \bar{G}$ for every element $h \in \overline{\mathbb{H}}$. Then $g \in \overline{\mathbb{H}}$.

Proof The required assertion follows from [17, Section 1].

Lemma A.5 Let $F$ be a proper normal subgroup of 2.A . Then either $F \cong \mathbb{Z}_{2}$ is a center of the group $2 . \mathrm{A}_{4}$, or $F \cong \mathbb{Q}_{8}$, where $\mathbb{Q}_{8}$ is the quaternion group of order 8 .

Proof The only nontrivial normal subgroup of the group $\mathrm{A}_{4}$ is isomorphic to the group $\mathbb{Z}_{2} \times \mathbb{Z}_{2}$.

Lemma A.6 The group $\overline{\mathbb{H}}$ contains no proper nontrivial subgroups that are normal in $\bar{G}$.

Proof Let $\theta: \overline{\mathbb{H M}} \rightarrow \operatorname{Aut}(\overline{\mathbb{H}})$ be the homomorphism such that

$$
\theta(g)(h)=g h g^{-1} \in \overline{\mathbb{H}}
$$

for all $g \in \overline{\mathbb{H M}}$ and $h \in \overline{\mathbb{H}}$. Then $\operatorname{ker}(\theta)=\overline{\mathbb{H}}$ by Lemma A.4.

The homomorphism $\theta$ induces an isomorphism $\tau: \mathbb{M} \rightarrow \operatorname{SL}_{2}\left(\mathbb{F}_{5}\right)$.

Let $F \subset \mathbb{M}$ be a subgroup such that $\beta(F)=\beta(G) \cong 2$. A $_{4}$. Then $G=\mathbb{H} \rtimes F$.

Suppose that the group $\overline{\mathbb{H}}$ contains a proper nontrivial subgroup that is a normal subgroup of the group $\bar{G}$. Let us consider $\overline{\mathbb{H}}$ as a two-dimensional vector space over $\mathbb{F}_{5}$. Then $\mathbb{F}_{5}^{2} \cong \overline{\mathbb{H}}=V_{0} \oplus V_{1}$, where $V_{0}$ and $V_{1}$ are one-dimensional $\tau(F)$ invariant subspaces, since $\left|2 . \mathrm{A}_{4}\right|=24$ is coprime to 5 .

By Lemma A.4, the homomorphism $\tau$ induces a monomorphism

$$
F \longrightarrow \mathrm{GL}_{1}\left(\mathbb{F}_{5}\right) \times \mathrm{GL}_{1}\left(\mathbb{F}_{5}\right) \cong \mathbb{Z}_{4} \times \mathbb{Z}_{4}
$$

which implies that $F$ is an abelian group, which is not the case.

Lemma A.7 The group $\bar{G}$ does not contain proper normal subgroups not containing $\overline{\mathbb{H}}$.

Proof Suppose that $\bar{G}$ contains a normal subgroup $\bar{G}^{\prime}$ such that $\overline{\mathbb{H}} \nsubseteq \bar{G}^{\prime}$. Then the intersection $\bar{G}^{\prime} \cap \overline{\mathbb{H}}$ consists of the identity element in $G$ by Lemma A.6. Hence

$$
\bar{G}^{\prime} \cong \beta\left(\bar{G}^{\prime}\right) \subseteq \beta(\bar{G}) \cong 2 . \mathrm{A}_{4},
$$

which implies that $\bar{G}^{\prime}$ is isomorphic to a normal subgroup of the group 2.A 4 .

Let $\bar{Z}$ be the center of $\bar{G}^{\prime}$. Then $\bar{Z}$ is a normal subgroup of the group $\bar{G}$. Thus, we have $\bar{Z} \cong \mathbb{Z}_{2}$ by Lemma A.5. Hence $\bar{Z}$ is contained in the center of $\bar{G}$, which contradicts Lemma A.4. 
Lemma A.8 Let $E$ be a smooth irreducible curve of genus $g \leqslant 8$. Then there is no monomorphism $\bar{G} \rightarrow \operatorname{Aut}(E)$.

Proof By classification of finite subgroups in $\mathrm{PGL}_{2}(\mathbb{C})$ the case $g=0$ is impossible. The cases $2 \leqslant g \leqslant 8$ are impossible by Theorem 2.14 . Therefore, we may assume that $E$ is an elliptic curve.

Let us consider $E$ as an abelian group. Then there is an exact sequence

$$
1 \longrightarrow E \stackrel{\iota}{\longrightarrow} \operatorname{Aut}(E) \stackrel{v}{\longrightarrow} \mathbb{Z}_{n} \longrightarrow 1
$$

for some $n \in\{2,4,6\}$.

Suppose that there is a monomorphism $\theta: \bar{G} \rightarrow \operatorname{Aut}(E)$. Then $\theta(\overline{\mathbb{H}}) \subset \iota(E)$, because $\iota(E)$ contains all the elements of $\operatorname{Aut}(E)$ of order 5 .

Let $g$ be any element of $\bar{G}$ such that $\theta(g) \in \iota(E)$. Then $\theta(g) \theta(h)=\theta(h) \theta(g)$ for every $h \in \overline{\mathbb{H}}$, because $\iota(E)$ is an abelian group, and thus $g \in \overline{\mathbb{H}}$ by Lemma A.4. Hence $\theta(\bar{G}) \cap \iota(E)=\theta(\overline{\mathbb{H}})$, which implies that $v(\bar{G}) \cong \beta(\bar{G}) \cong 2 . \mathrm{A}_{4}$, which is absurd.

The main purpose of this section is to prove the following result.

Theorem A.9 Let $E$ be a smooth elliptic curve. Then there is no exact sequence of groups

$$
1 \longrightarrow G^{\prime} \stackrel{\iota}{\longrightarrow} \bar{G} \stackrel{v}{\longrightarrow} G^{\prime \prime} \longrightarrow 1
$$

where $G^{\prime}$ and $G^{\prime \prime}$ are subgroups of the groups $\operatorname{Aut}\left(\mathbb{P}^{1}\right)$ and $\operatorname{Aut}(E)$, respectively.

Proof Suppose that the exact sequence of groups (A.10) does exist. Then $\iota$ is not an isomorphism, because the group $\operatorname{Aut}\left(\mathbb{P}^{1}\right)$ does not contain subgroups isomorphic to $\bar{G}$. The monomorphism $v$ is not an isomorphism by Lemma A.8. Then $\overline{\mathbb{H}} \subset \iota\left(G^{\prime}\right)$ by Lemma A.7. But Aut $\left(\mathbb{P}^{1}\right)$ contains no subgroups isomorphic to $\overline{\mathbb{H}}$, which is a contradiction.

Corollary A.11 There is no monomorphism $\bar{G} \rightarrow \operatorname{Bir}\left(E \times \mathbb{P}^{1}\right)$, where $E$ is a smooth elliptic curve.

We believe that there is a simpler proof of Theorem A.9. 


\section{References}

[1] A Adler, On the automorphism group of a certain cubic threefold, Amer. J. Math. 100 (1978) 1275-1280 MR522700

[2] C Birkar, P Cascini, CD Hacon, J McKernan, Existence of minimal models for varieties of log general type, J. Amer. Math. Soc. 23 (2010) 405-468 MR2601039

[3] H F Blichfeldt, Finite collineation groups, Univ. of Chicago Science Series, Univ. of Chicago Press (1917)

[4] C P Boyer, K Galicki, J Kollár, Einstein metrics on spheres, Ann. of Math. (2) 162 (2005) 557-580 MR2178969

[5] R Brauer, Über endliche lineare Gruppen von Primzahlgrad, Math. Ann. 169 (1967) 73-96 MR0206088 In German

[6] T Breuer, Characters and automorphism groups of compact Riemann surfaces, London Math. Soc. Lecture Note Series 280, Cambridge Univ. Press (2000) MR1796706

[7] H Burkhardt, Untersuchungen aus dem Gebiete der hyperelliptischen Modulfunctionen, Math. Ann. 38 (1891) 161-224 MR1510670 In German

[8] H D Cao, Deformation of Kähler metrics to Kähler-Einstein metrics on compact Kähler manifolds, Invent. Math. 81 (1985) 359-372 MR799272

[9] I Cheltsov, Log canonical thresholds of del Pezzo surfaces, Geom. Funct. Anal. 18 (2008) 1118-1144 MR2465686

[10] I Cheltsov, K Shramov, Six-dimensional exceptional quotient singularities arXiv: 1001.3863

[11] I Cheltsov, K Shramov, Log-canonical thresholds for nonsingular Fano threefolds, Russian Math. Surveys 63 (2008) 859-958 MR2484031

[12] M J Collins, On Jordan's theorem for complex linear groups, J. Group Theory 10 (2007) 411-423 MR2334748

[13] J H Conway, R T Curtis, S P Norton, R A Parker, R A Wilson, Atlas of finite groups: Maximal subgroups and ordinary characters for simple groups, Oxford Univ. Press, Eynsham (1985) MR827219 With computational assistance from J G Thackray

[14] W Feit, The current situation in the theory of finite simple groups, from: "Actes du Congrès International des Mathématiciens (Nice, 1970), Tome 1", Gauthier-Villars, Paris (1971) 55-93 MR0427449

[15] W Feit, J G Thompson, Groups which have a faithful representation of degree less than ( $p-1 / 2)$, Pacific J. Math. 11 (1961) 1257-1262 MR0133373

[16] F Hidaka, K Watanabe, Normal Gorenstein surfaces with ample anti-canonical divisor, Tokyo J. Math. 4 (1981) 319-330 MR646042

[17] G Horrocks, D Mumford, A rank 2 vector bundle on $\mathbf{P}^{4}$ with 15, 000 symmetries, Topology 12 (1973) 63-81 MR0382279 
[18] S Ishii, Y Prokhorov, Hypersurface exceptional singularities, Internat. J. Math. 12 (2001) 661-687 MR1875648

[19] V A Iskovskikh, Y G Prokhorov, Fano varieties, from: "Algebraic geometry, V", Encyclopaedia Math. Sci. 47, Springer, Berlin (1999) 1-247 MR1668579

[20] J M Johnson, J Kollár, Fano hypersurfaces in weighted projective 4-spaces, Experiment. Math. 10 (2001) 151-158 MR1822861

[21] Y Kawamata, On Fujita's freeness conjecture for 3-folds and 4-folds, Math. Ann. 308 (1997) 491-505 MR1457742

[22] Y Kawamata, Subadjunction of log canonical divisors, II, Amer. J. Math. 120 (1998) 893-899 MR1646046

[23] J Kollár, Singularities of pairs, from: "Algebraic geometry-Santa Cruz 1995", (J Kollár, R Lazarsfeld, D R Morrison, editors), Proc. Sympos. Pure Math. 62, Amer. Math. Soc. (1997) 221-287 MR1492525

[24] S A Kudryavtsev, On purely log terminal blow-ups, Math. Notes 69 (2002) 814-819 MR1861570

[25] R Lazarsfeld, Positivity in algebraic geometry II: Positivity for vector bundles, and multiplier ideals, Ergebnisse der Math. und ihrer Grenzgebiete. 3. Folge. A Series of Modern Surveys in Math. 49, Springer, Berlin (2004) MR2095472

[26] C L Mallows, N A Sloane, On the invariants of a linear group of order 336, Proc. Cambridge Philos. Soc. 74 (1973) 435-440 MR0325607

[27] D Markushevich, Y G Prokhorov, Exceptional quotient singularities, Amer. J. Math. 121 (1999) 1179-1189 MR1719826

[28] H Maschke, Aufstellung des vollen Formensystems einer quaternären Gruppe von 51840 linearen Substitutionen, Math. Ann. 33 (1889) 317-344 MR1510546 In German

[29] J Nagura, On the interval containing at least one prime number, Proc. Japan Acad. 28 (1952) 177-181 MR0050615

[30] I Nieto, The normalizer of the level $(2,2)$-Heisenberg group, Manuscripta Math. 76 (1992) 257-267 MR1185019

[31] Y G Prokhorov, Blow-ups of canonical singularities, from: "Algebra (Moscow, 1998)", (Y Bahturin, editor), de Gruyter, Berlin (2000) 301-317 MR1754677

[32] Y G Prokhorov, Boundedness of exceptional quotient singularities, Math. Notes 68 (2000) 664-667 MR1835460

[33] Y G Prokhorov, Fields of invariants of finite linear groups, from: "Cohomological and geometric approaches to rationality problems”, (F Bogomolov, Y Tschinkel, editors), Progr. Math. 282, Birkhäuser, Boston (2010) 245-273 MR2605172

[34] S Ramanujan, A proof of Bertrand's postulate, J. Indian Math. Soc. 11 (1919) 181-182 MR2280867 
[35] Y A Rubinstein, Some discretizations of geometric evolution equations and the Ricci iteration on the space of Kähler metrics, Adv. Math. 218 (2008) 1526-1565 MR2419932

[36] D Sakovics, Weakly-exceptional quotient singularities arXiv:1006.5909

[37] G C Shephard, J A Todd, Finite unitary reflection groups, Canadian J. Math. 6 (1954) 274-304 MR0059914

[38] V V Shokurov, Three-fold log flips, Russian Acad. Sci. Izv. Math. 40 (1993) 95-202 MR1162635

[39] V V Shokurov, Complements on surfaces, J. Math. Sci. (New York) 102 (2000) 38763932 MR1794169

[40] T A Springer, Invariant theory, Lecture Notes in Math. 585, Springer, Berlin (1977) MR0447428

[41] J G Thompson, Invariants of finite groups, J. Algebra 69 (1981) 143-145 MR613863

[42] G Tian, On Kähler-Einstein metrics on certain Kähler manifolds with $C_{1}(M)>0$, Invent. Math. 89 (1987) 225-246 MR894378

[43] G Tian, On a set of polarized Kähler metrics on algebraic manifolds, J. Differential Geom. 32 (1990) 99-130 MR1064867

[44] G Tian, S-T Yau, Kähler-Einstein metrics on complex surfaces with $C_{1}>0$, Comm. Math. Phys. 112 (1987) 175-203 MR904143

[45] G Tian, X Zhu, Convergence of Kähler-Ricci flow, J. Amer. Math. Soc. 20 (2007) 675-699 MR2291916

[46] S T Yau, On the Ricci curvature of a compact Kähler manifold and the complex MongeAmpère equation I, Comm. Pure Appl. Math. 31 (1978) 339-411 MR480350

[47] S S-T Yau, Y Yu, Gorenstein quotient singularities in dimension three, Mem. Amer. Math. Soc. 105, no. 505, Amer. Math. Soc. (1993) MR1169227

School of Mathematics, University of Edinburgh

James Clerk Maxwell Building, Mayfield Road, Edinburgh, EH93JZ, UK

Steklov Institute of Mathematics

8 Gubkina street, Moscow, 119991, Russia

IC, CS: Laboratory of Algebraic Geometry, GU-HSE

7 Vavilova street, Moscow 117312, Russia

i.cheltsov@ed.ac.uk, shramov@mccme.ru

http://www.maths.ed.ac.uk/cheltsov/

Proposed: Gang Tian

Seconded: Simon Donaldson, Richard Thomas
Received: 28 October 2009

Revised: 20 June 2011 